ROM2F-94-17

July 7,2021

\title{
ALE Instantons in String Effective Theory
}

\author{
M. Bianchi, F. Fucito, G.C. Rossi \\ Dipartimento di Fisica, Università di Roma II "Tor Vergata" \\ I.N.F.N. Sezione di Roma II "Tor Vergata",00133 Roma, Italy \\ and \\ M. MARTELLini \\ Dipartimento di Fisica, Università di Milano, 20133 Milano, Italy \\ Sezione I.N.F.N. dell'Università di Pavia, 27100 Pavia, Italy
}

\begin{abstract}
We show that the classical equations of motion of the low-energy effective field theory describing the massless modes of the heterotic (or type I) string admit two classes of supersymmetric self-dual backgrounds. The first class, which was already considered in the literature, consists of solutions with a (conformally) flat metric coupled to axionic instantons. The second includes Asymptotically Locally Euclidean (ALE) gravitational instantonic backgrounds coupled to gauge instantons through the so-called "standard embedding". We show that some elements of these two classes of solutions are dual to each other in the sense of Buscher's duality. We give a world-sheet interpretation of the heterotic ALE istanton solutions in terms of superconformal $N=(4,4) \sigma$-models and argue for their validity to all orders in $\alpha^{\prime}$. Specializing the gravitational background to the Eguchi-Hanson instanton, we compute the indices of the fermionic operators and give the explicit form of all the relevant fermionic and bosonic zero-modes.
\end{abstract}




\section{Introduction}

Several non trivial solutions of general relativity (black holes, plane gravitational waves, cosmological metrics, ...) have been shown to be also classical solutions of some string theory. This is usually proven by recognizing that the one loop $\beta$-functions of the associated non-linear $\sigma$-models, describing the propagation of the string in the corresponding backgrounds, are zero. In turn these conditions can be looked at as the Euler-Lagrange equations of an effective action describing the low energy interactions of the massless modes of the string $[1,2,3]$.

As is well known, in order to find classical solutions of a supersymmetric theory, it is enough to set to zero the fermion fields together with their supersymmetric variations. This is the way in which several monopole [4], soliton [5] and instanton [6] configurations have been proven to be classical solutions (known as "pentabranes" [7]) of the effective supergravity theory derived from the heterotic string. In these solutions the metric is conformally flat.

The $\sigma$-models describing the propagation of the heterotic string in these backgrounds have $N=(4,0)$ supersymmetry on the world-sheet. If the gauge connection is identified with the generalized spin connection with torsion ("standard embedding"), the resulting $\sigma$-model turns out to be left-right symmetric and the $N=(4,0)$ supersymmetry is enhanced to $N=(4,4)$. As a consequence, all $\alpha^{\prime}$ corrections to the $\beta$-functions are expected to be zero [8].

In particular the instantonic solution of [6] (in the limit in which the dilaton field is taken to vanish at infinity) was proven to be an exact solution of the heterotic string equations of motion by showing that the corresponding $\sigma$-model coincides with an exactly conformal WZW model on the group manifold $S U(2)_{k} \otimes U(1)_{Q}$, where the level $k$ and the background charge $Q$ are related by $Q=1 / \sqrt{k+2}$. With this choice the coefficient of the conformal anomaly turns out to be $c=6$, as in the case of four flat supercoordinates.

Generally speaking, all higher order $\alpha^{\prime}$ corrections to the heterotic string classical solutions appear to be proportional to $\operatorname{tr} R \wedge R-\operatorname{tr} F \wedge F$ (or contractions 
thereof) [9], which indeed vanish thanks to the identification of $R$ with $F$, following from the "standard embedding". This observation has been widely used to derive consistent compactifications of the heterotic string on compact Calabi-Yau manifolds [10].

It is curious to note in this context that, to the extent of our knowledge, the first example of use of the "standard embedding" was to generate Yang-Mills instantons out of gravitational ones [11] in a Yang-Mills field theory coupled to gravity.

In this paper we will consider the possibility of promoting the solutions of the Euclidean Einstein equations, known as gravitational instantons, to fullfledged solutions of the heterotic string classical equations of motion. We will mostly concentrate our attention on gravitational instantons which correspond to non-compact, four-dimensional Asymptotically Locally Euclidean (ALE) manifolds admitting Ricci-flat hyperkähler metrics with self-dual curvature.

To construct a solution of the whole set of classical equations we notice that ALE instantons satisfy the empty space Euclidean Einstein equations. Thus in order to couple them to non-trivial backgrounds of the other massless bosonic fields, one must consider field configurations with zero Euclidean stress energy tensor. Gauge instantons and axionic instantons [12] are solutions of the equations of motion in the absence of fermionic sources, which precisely meet this requirement.

For the consistency of the solution the validity of the (modified) Bianchi identities has to be imposed. To fulfill this requirement one is naturally led, as a first step, to identify the gauge and the (generalized) spin connection. In this way the Bianchi identities are simply reduced to the requirement that the dilaton field must be a constant or the metric conformally flat.

It is remarkable that, as we will show in sect. 2.2, the solution in which the gravitational background is a self-dual multi-center metric and the dilaton field is a constant, turns out to be dual, in the sense of Buscher's duality [13], to the solution found in [4], in which, viceversa, the gravitational background is conformally flat and the whole non triviality lies in the form of the dilaton. As for the solution 
discussed in [6], its dual is the stringy black hole solution described in [14].

Many topological properties of ALE manifolds have been known for a while. The possibility of finding new topological invariants, through the study of topological quantum field theories [15] on such manifolds, may not forego the precise determination of the indices of the fermionic operators and of all the relevant bosonic and fermionic zero-modes. The computations can be drastically simplified by exploiting the existence of a surviving global $N=1$ right-handed supersymmetry, related to the presence of two independent covariantly constant spinors [16]. This supersymmetry allows to relate zero-modes of the bosonic fields to those of their fermionic superpartners. In this way one is reduced to compute fermionic zero-modes only.

In order to be explicit we shall take the simplest among the ALE manifolds: the Eguchi-Hanson instanton [17] and we complete the solution of the classical equations of motion by coupling it to a gauge instanton constructed by identifying the gauge connection with the EH spin connection (standard embedding). As far as the classical invariants of the manifold are concerned, i.e. Euler characteristic, Hirzebruch signature, indices of the Dirac and Rarita-Schwinger operators in the gauge singlet representation, the relevant computations can be found in the classical literature on the subject [18].

New topological invariants are related to the indices of the Dirac operator in non-singlet representations of the gauge group. We show that the number of (left-handed) zero-modes of the Dirac operator for spinors in the fundamental representation of the instantonic $S U(2)$ gauge group is 1, while for spinors in the adjoint representation this number is 6 . The values taken by these two indices are respectively related to the existence on this background of 1 self-dual closed two-form and of 12 deformations of the tangent bundle preserving the rank and the condition of vanishing of the first Chern class. The parameters associated with these deformations lend themselves to an interpretation as collective coordinates of the gauge connection. 
The explicit form of all zero-modes is found both with the help of geometrical intuition and/or by solving directly, when necessary, the Dirac equation.

The relevance of the properties of the classical solutions of a field theory and of the explicit form of the zero-modes of the wave operators around the corresponding backgrounds needs no further stressing here, if not to recall that any non-perturbative instantonic saddle-point evaluation of an Euclidean functional integral would require a detailed knowledge of all these ingredients [19, 20].

In the case of global supersymmetric theories a detailed study of a possible breaking mechanism of SUSY via gauge instantons was carried out in [21] with rather encouraging results. The case of locally supersymmetric theories was addressed to in $[16,22]$, where it was argued that, in supergravity, gravitino condensation due to gravitational instantons can trigger the breaking of SUSY. An important step forward in this line of arguments was made in [23], where, in the case of $N=1$ supergravity, the gravitino field strength condensate, $\left\langle\psi_{a b} \psi^{a b}>\right.$, was indeed computed and shown to be finite and space-time independent. The calculation was done by performing a saddle-point approximation around the non trivial classical solution of the theory represented by the Eguchi-Hanson gravitational instanton [18].

The idea behind this kind of approach is the hope of being able to infer SUSY breaking by putting non trivial condensates in relation with some anomalous SUSY transformation. In global SUSY theories in flat space [21] such a relation exists and it is called the Konishi anomaly equation [24]. For a Super Yang-Mills theory coupled to chiral scalar matter multiplets, $\Phi^{i}$, belonging to the representations $\underline{R}^{i}$ of the gauge group, the relevant anomalous commutator reads

$$
\left\{\bar{Q}, \bar{\lambda}_{i} \phi^{j}\right\}=\frac{q^{2} c_{i}}{32 \pi^{2}} \chi \chi \delta_{i}^{j}+\phi^{j} \frac{d \bar{W}}{d \bar{\phi}_{i}}
$$

where $i, j$ are flavour indices, $W$ is the superpotential, $\bar{Q}$ is a SUSY charge, $q$ the gauge coupling constant, $\chi$ is the gaugino, $\phi^{i}$ and $\lambda^{i}$ are components of the chiral 
multiplet, $\Phi^{i}$, and $c_{i}$ is the index of the representation $\underline{R}_{i}$. The anomaly equation (1.1) lies in the same supermultiplet as the chiral anomaly, i.e. it is a partner of the anomalous divergence equation of the $R$-symmetry current of the theory. The discovery that (some of) the condensates appearing in (1.1) acquire a non-zero vacuum expectation value allowed to derive detailed information on the degeneracy pattern of the vacuum state manifold and, in some cases (SUSY theories with no flat directions and chiral matter in suitably choosen representations of the gauge group) even to conclude that SUSY is dynamically broken by non-perturbative instanton effects.

To construct a similar argument in supergravity one has to start with the anomalous divergence of the $R$-symmetry chiral current, $j^{\mu}$

$$
D_{\mu} j^{\mu}=-\frac{1}{384 \pi^{2}} R_{a b c d} \widetilde{R}^{a b c d}
$$

where the tilde stands for the duality operation. Since $R_{a b c d} \widetilde{R}^{a b c d}$ is the top component of a chiral multiplet, which has $\psi_{a b} \psi^{a b}$ as lowest component [25], the analogue of the anomalous SUSY transformation (1.1) is

$$
\{\bar{Q}, \bar{\lambda} \phi\}=\frac{\kappa^{2}}{384 \pi^{2}} \psi_{a b} \psi^{a b}
$$

where $\kappa$ is the gravitational coupling constant, $\psi^{a b}$ is the gravitino field-strength and $\lambda$ and $\phi$ are components of a chiral matter multiplet (for the sake of definiteness they may be respectively taken to be the dilatino and the dilaton). The appearance of the gravitino field-strength bilinear in the right-hand-side of (1.3) led correctly the authors of [23] to compute the expectation value of $\psi_{a b} \psi^{a b}$, instead of $\psi_{\mu} \psi^{\mu}$, as suggested in [16].

Many of the computations we will present in this paper are very much in the same line of thought of instanton calculus in global supersymmetric theories [21], where, as we said, the simultaneous presence of global supersymmetry and of classical instantonic solutions conspire to give exact space-time independent constant 
results for certain correlators. The calculations we present here are propaedeutical to extend instanton calculus to locally supersymmetric (i.e. to supergravity) theories $[26,27]$.

Contrary to globally supersymmetric Yang-Mills theories, however, supergravity is not renormalizable. Strictly speaking, this puts the entire subject of instanton calculus in supergravity on a rather shaky basis. On this problem we would like to take the point of view (as also suggested in [23]) that supergravity theories should be considered as low energy limits of string theories, which are expected not to suffer from these deficencies. Thus to the order to which supergravity theories are formally renormalizable (i.e. generically up to two loops) results from perturbative and non-perturbative (instanton) calculations should be considered as the limiting values of the corresponding exact string results.

There are two more reasons to pursue this philosophy we would like to mention here. The first has to do with the fact that effective field theories appear at the moment the only arena where non-perturbative aspects of string theories can be studied. The second is that, exploiting the relation between bosonic zero-modes and instanton "collective coordinates", explicit computations of the former may prove to be a useful starting point in the investigation of the structure of the istanton moduli space over non-compact ALE manifolds. Except for the purely gravitational sector, this subject is as yet poorly understood.

The plan of the paper is as follows. In section 2.1 we show that the effective supergravity theory arising from the heterotic string admits classical solutions in which the gravitational background is a self-dual ALE metric and the corresponding gauge background is constructed by the identifying the gauge connection with the Levi-Civita spin connection ("standard embedding"). In the following we will generically refer to these solutions as "heterotic ALE instantons". In section 2.2 we give a world-sheet interpretation of these classical solutions and we study the relevant Buscher's duality transformations, showing that heterotic multicenter instantons are dual to the conformally flat solitonic solutions discovered in [4]. 
In section 2.3 we specialize the self-dual ALE metric to the Eguchi-Hanson (EH) gravitational instanton and we collect various useful formulae for the coupled gauge and gravitational background obtained in this particular case. In the following we will call this solution the "heterotic EH instanton". In section 3 we compute the indices of the gaugino Dirac operator in the heterotic EH background with the gaugino in the fundamental and the adjoint representation of the gauge group. In section 4 we determine the explicit form of the zero-modes of the operators of the small fluctuations around the heterotic EH background for all the massless fields of the theory and we discuss the relation between zero-modes and instanton collective coordinates. Section 5 contains our conclusions and an outlook of future lines of investigation. In Appendix A we state our notations and we gather a series of definitions which should allow the interested reader to reproduce our results. In Appendix B we report some known facts about the geometry of four-dimensional ALE manifolds with a special emphasis on the properties which may be of interest for the study of string propagation on these backgrounds or for the determination of the structure of their moduli space.

\section{Gravitational instantons in heterotic string effective theories}

\subsection{General Form of Heterotic AlE Istantons}

Our starting point is the $D=10, N=1$ action that arises as a low-energy effective field theory from the heterotic (or type I) string. For the massless bosonic fields of the theory (graviton $g_{M N}$, dilaton $\phi$, antisymmetric tensor $b_{M N}$, vector bosons $A_{M}$ ) the Lagrangian takes the form

$$
L_{B}=\sqrt{g}\left\{-\frac{1}{2 \kappa^{2}} R(g)-\frac{1}{4 \kappa^{2}}(\partial \phi)^{2}-\frac{e^{-\phi}}{12 \kappa^{2}} H^{2}-\frac{e^{-\frac{\phi}{2}}}{4 q^{2}} \operatorname{tr}\left(F^{2}\right)\right\}
$$

The part of the lagrangian quadratic in the fermionic fields (gravitino $\psi_{M}$, dilatino 
$\lambda$, gaugino $\chi)$ is given by

$$
\begin{aligned}
L_{F} & =\sqrt{g}\left\{-\frac{1}{2} \bar{\psi}_{M} \Gamma^{M N P} D_{N} \psi_{P}-\frac{1}{2} \bar{\lambda} \Gamma^{M} D_{M} \lambda-\frac{1}{2} \operatorname{tr}\left(\bar{\chi} \Gamma^{M} D_{M} \chi\right)\right. \\
& -\frac{1}{2 \sqrt{2}} \bar{\psi}_{M} \Gamma^{N} \Gamma^{M} \lambda \partial_{N} \phi+\frac{\sqrt{2} e^{-\frac{\phi}{2}}}{48} \operatorname{tr}\left(\bar{\chi} \Gamma^{M N P} \chi\right) H_{M N P} \\
& -\frac{\kappa e^{-\frac{\phi}{4}}}{4 q} \operatorname{tr}\left(\bar{\chi} \Gamma^{M} \Gamma^{N P} F_{N P}\right)\left(\psi_{M}+\frac{\sqrt{2}}{12} \Gamma_{M} \lambda\right)+\frac{e^{-\frac{\phi}{2}} \sqrt{2}}{48} \\
& \left.\left(\bar{\psi}_{M} \Gamma^{M N P Q R} \psi_{R}+6 \bar{\psi}^{N} \Gamma^{P} \psi^{Q}-\sqrt{2} \bar{\psi}_{M} \Gamma^{N P Q} \Gamma^{M} \lambda\right) H_{N P Q}\right\}
\end{aligned}
$$

In (2.1) and (2.2) $\kappa$ and $q$ are respectively the gravitational and the gauge coupling constants. $g$ is the determinant of the metric, $R$ is the curvature scalar and $F$ is the Yang-Mills field-strength. $H$ is the field-strength of the antisymmetric tensor, modified by the addition of the Chern-Simons three-form [28]

$$
\begin{aligned}
H & =d b-\frac{\kappa^{2}}{q^{2}} \Sigma_{Y M} \\
\Sigma_{Y M} & =\operatorname{Tr}\left(A \wedge d A+\frac{2}{3} A \wedge A \wedge A\right)
\end{aligned}
$$

This modification is needed to ensure the invariance of the full action (including four-fermion terms which for short have not been displayed above) under local supersymmetry transformations.

It should be noted, however, that in order to implement the Green-Schwarz mechanism of anomaly cancellation the standard way to proceed [1] is to add to the definition of $H$ the Chern-Simons three-form for the Lorentz group ${ }^{\star}$

$$
\Sigma_{L}=\operatorname{tr}\left(\omega \wedge d \omega+\frac{1}{3} \omega \wedge \omega \wedge \omega\right)
$$

where $\omega$ is the Levi-Civita spin connection. This redefinition triggers a chain of further modifications of the Lagrangian and of supersymmetry transformations which

\footnotetext{
^ By Tr we mean trace in the adjoint representation of the gauge group. By tr we mean trace in the vector representation of the Lorentz group
} 
have been partly carried out in [9]. In particular the authors of [9] have shown that to first order in $\kappa^{2}$ it is necessary to add to the Lagrangian a term quadratic in the Riemann tensor. Here one has the well known ambiguity related to the possibility of adding terms proportional to the square of the Ricci tensor or to the curvature scalar which vanish by the lowest order equations of motion. Our strategy will be to solve to lowest order in $\alpha^{\prime}$ the equations of motion, but to include the ChernSimons Lorentz three-form in the definition of $H$, when we require the validity of the Bianchi identities, for the sake of showing that certain supersymmetric configurations are in fact consistent solutions of the classical equations of motion to all orders in $\alpha^{\prime}$.

In $D=10$, the relation among the inverse string tension $\alpha^{\prime}$ (with mass dimension -2 ), the gravitational constant $\kappa$ (with mass dimension -4 ) and the gauge coupling $q$ (with mass dimension -3 ) is $\kappa^{2}=q^{2} \alpha^{\prime}$ for the heterotic string (or $\kappa=\frac{q^{2}}{\alpha^{\prime}}$ for the type I superstring). As a result, in (2.1) the $q$-dependence can be factorized out and reabsorbed into a redefinition of the dilaton factor, after a Weyl rescaling of the metric. Eventually the (vacuum expectation value of the) dilaton factor will play the role of string loop expansion parameter [29].

The procedure described above leads to the so-called $\sigma$-model variables which in $D$ dimensions are related to the canonical ones by the equations (capital letters stand for $\sigma$-model variables)

$$
\begin{aligned}
G_{M N} & =e^{\frac{4 \phi}{D-2}} g_{M N}, \quad B_{M N}=b_{M N}, \quad A_{M}=A_{M} \\
\Psi_{M} & =e^{\frac{\phi}{D-2}}\left(\psi_{M}-\frac{\Gamma_{M}}{2 \sqrt{2}} \lambda\right) \quad \Lambda=e^{\frac{\phi}{D-2}} \lambda \quad X=e^{\frac{\phi}{D-2}} \chi
\end{aligned}
$$

In terms of the $\sigma$-model variables the bosonic part of the lagrangian becomes [2]

$$
\mathcal{L}_{B}=\frac{\sqrt{G}}{2 \alpha^{\prime}} e^{-2 \phi}\left(R-4(\partial \phi)^{2}+\frac{1}{12} H^{2}+\frac{\alpha^{\prime}}{2} F^{2}\right)
$$

where $G$ is the determinant of the metric $G_{M N}$. The Euler-Lagrange equations 
obtained by varying $(2.6)$ are

$$
\begin{aligned}
R_{M N}-\frac{1}{2} G_{M N} R & =\alpha^{\prime} T_{M N} \\
D_{M}\left(e^{-2 \phi} F^{M N}\right) & =A_{M} D_{P}\left(e^{-2 \phi} H^{M N P}\right) \\
D_{M} D^{M} \phi+\frac{1}{6} e^{-2 \phi} H^{2} & =\alpha^{\prime}\left\{F(A)^{2}-R\left(\Omega_{-}\right)^{2}\right\} \\
D_{M}\left(e^{-2 \phi} H^{M N P}\right) & =0
\end{aligned}
$$

where $T_{M N}$ is the total stress energy tensor and $D_{M}$ the fully covariant derivative. In presence of a non-zero torsion one can define the two generalized spin connections

$$
\Omega_{M \pm}^{A B}=\omega_{M}^{A B} \pm H_{M}^{A B}
$$

where $A, B$ are frame indices. Note that it is the generalized spin connection $\Omega_{-}$, which enters in (2.7).

We look for a supersymmetric solution of the above equations whose instantaneous time slice is five-dimensional [7]. We split the 10 dimensional coordinates $\left\{x^{M}\right\}$ in two sets: a six-dimensional one $\left\{y^{i} ; i=0,1, \cdots, 5\right\}$ and a fourdimensional Euclidean part $\left\{x^{\mu} ; \mu=0,1,2,3\right\}^{\star}$. We assume that the fields do not depend on the $y$-coordinates and that the only non-trivial components of all tensors are those spanning the four-dimensional transverse space. With this restriction the sum of the dilaton, axion and vector boson euclidean stress energy tensor becomes

$$
\begin{aligned}
T_{\mu \nu} & =\frac{1}{4} G^{\rho \sigma}\left(F_{\mu \rho}+\widetilde{F}_{\mu \rho}\right)\left(F_{\nu \sigma}-\widetilde{F}_{\nu \sigma}\right)-\frac{1}{8} G_{\mu \nu}\left((\partial \phi)^{2}-(\widetilde{H})^{2}\right) \\
& +\frac{1}{2 \alpha^{\prime}}\left(\partial_{\mu} \phi+\widetilde{H}_{\mu}\right)\left(\partial_{\nu} \phi-\widetilde{H}_{\nu}\right)+\frac{1}{2 \alpha^{\prime}}\left(\partial_{\mu} \phi-\widetilde{H}_{\mu}\right)\left(\partial_{\nu} \phi+\widetilde{H}_{\nu}\right)
\end{aligned}
$$

where the tilde indicates the dual of the field-strengths $F$ and $H$.

\footnotetext{
$\star$ In spite of the fact that throughout this paper we will stick to four-dimensional metrics with Euclidean signature, we will label space-time coordinates with the index $\mu$ running from 0 to 3
} 
An ansatz for the solution of the bosonic equations of motion, for which the stress energy tensor (2.9) vanishes, is given by $[6,4,7]$

$$
\begin{aligned}
F_{\mu \nu} & = \pm \widetilde{F}_{\mu \nu}= \pm \frac{1}{2} \sqrt{G} \varepsilon_{\mu \nu}{ }^{\rho \sigma} F_{\rho \sigma} \\
G_{\mu \nu} & =e^{2 \phi} \widehat{g}_{\mu \nu} \\
H_{\mu \nu \rho} & = \pm \sqrt{G} \varepsilon_{\mu \nu \rho} \sigma \partial_{\sigma} \phi
\end{aligned}
$$

with $\widehat{g}_{\mu \nu}$ any arbitrary Ricci flat metric. The fermionic equations of motion are easily satisfied by setting all fermion fields equal to zero $[1,10]$.

In order to find under which conditions this solution is supersymmetric, we observe that the local SUSY variations of the bosonic fields are all trivially zero, due to the above vanishing of the background fermion fields, while for the gravitino, dilatino and gaugino field variations one has

$$
\begin{aligned}
\delta \psi_{\mu} & =\left(\partial_{\mu}-\frac{1}{4} \Omega_{\mu+}{ }^{a b} \gamma_{a b}\right) \epsilon+\alpha^{\prime} \delta_{2} \psi_{\mu} \\
\delta \lambda & =-\frac{1}{4}\left(\gamma^{\mu} \partial_{\mu} \phi-\frac{1}{6} H_{\mu \nu \rho} \gamma^{\mu \nu \rho}\right) \epsilon+\alpha^{\prime} \delta_{2} \lambda \\
\delta \chi & =-\frac{1}{4} F_{\mu \nu} \gamma^{\mu \nu} \epsilon+\alpha^{\prime} \delta_{2} \chi
\end{aligned}
$$

where $\gamma_{\mu \nu} \ldots$ are the usual antisymmetric products of gamma matrices (see Appendix A). Greek and latin letters denote four-dimensional coordinates and frame indices respectively. In the background we are considering the parts of the variations indicated by $\delta_{2}$, being quadratic in the fermi fields, are all zero. It is also easy to see that, if we take the plus (minus) sign in (2.10) and a right-(left-)handed spinor, $\epsilon$, the variation of the gaugino is zero, as $F_{\mu \nu}$ is (anti)self-dual. Similarly the dilatino variation is zero, thanks to the ansatz for $H_{\mu \nu \rho}$. The generalized spin connections (with torsion) of the metric $G_{\mu \nu}$ become

$$
\Omega_{\mu \pm}^{a b}=\widehat{\omega}_{\mu}^{a b}-\left(\widehat{e}_{\mu}^{a} \widehat{e}^{b \nu}-\widehat{e}^{a \nu} \widehat{e}_{\mu}^{b}\right) \partial_{\nu} \phi \pm H_{\mu}^{a b} \equiv \widehat{\omega}_{\mu}^{a b}+w_{\mu}^{a b} \pm H_{\mu}^{a b}
$$

where hatted quantities are computed with respect to the metric $\widehat{g}_{\mu \nu}$. Using the duality relation $\frac{1}{2} \varepsilon^{a b}{ }_{c d} w_{\mu}^{c d}=H_{\mu}{ }^{a b}\left(\frac{1}{2} \varepsilon^{a b}{ }_{c d} w_{\mu}{ }^{c d}=-H_{\mu}{ }^{a b}\right)$, which again is a consequence of the ansatz (2.10), we see that the gravitino variation may be set to zero 
if $\widehat{\omega}_{\mu}^{a b}$ is taken to be (anti)self-dual. With this choice $\Omega_{\mu+}{ }^{a b}$ is (anti)self-dual, while $\Omega_{\mu-}{ }^{a b}$ has in general no definite duality. From now on we will specialize to the self-dual case by choosing the + sign in (2.10) and $\epsilon$ right-handed in (2.11).

To have a consistent supersymmetric solution, we must require the expressions (2.10) for $F$ and $H$ to be the field-strengths of the fundamental bosonic fields, $A_{\mu}$ and $B_{\mu \nu}$ respectively. This amounts to impose the validity of the (modified) Bianchi identities

$$
\begin{aligned}
& d F+A \wedge F=0 \\
& d H=\alpha^{\prime}\left\{\operatorname{tr} R\left(\Omega_{-}\right) \wedge R\left(\Omega_{-}\right)-\frac{1}{30} \operatorname{Tr} F(A) \wedge F(A)\right\}
\end{aligned}
$$

where, as we said, in $d H$ we also have included the contribution of the ChernSimons Lorentz three-form. To satisfy (2.13) it is natural to relate the gauge and spin connection through a generalized form of "standard embedding".

Given the existence of a self-dual and an antiself-dual 't Hooft symbol, $\eta_{a b}^{i}$ and $\bar{\eta}_{a b}^{i}$, and of two generalized spin connections, $\Omega_{ \pm}$, four gauge connections could in principle be constructed, namely

$$
\begin{aligned}
A^{i}{ }_{\mu} & =\frac{1}{2} \eta^{i}{ }_{a b} \Omega_{\mu-}{ }^{a b}=\frac{1}{2} \eta^{i}{ }_{a b} \widehat{\omega}_{\mu}{ }^{a b} \\
A^{i}{ }_{\mu} & =\frac{1}{2} \bar{\eta}^{i}{ }_{a b} \Omega_{\mu-}{ }^{a b}=\frac{1}{2} \bar{\eta}^{i}{ }_{a b}\left(w_{\mu}{ }^{a b}-H_{\mu}{ }^{a b}\right) \\
A^{i}{ }_{\mu} & =\frac{1}{2} \eta^{i}{ }_{a b} \Omega_{\mu+}{ }^{a b} \\
A^{i}{ }_{\mu} & =\frac{1}{2} \bar{\eta}^{i}{ }_{a b} \Omega_{\mu+}{ }^{a b}=0
\end{aligned}
$$

with $i=1,2,3$ the index of an $S U(2)$ subgroup of the heterotic gauge group.

Of the above four cases, the last one, (2.17), is trivial, because $\bar{\eta}_{a b}^{i}$ and $\Omega_{+}$ have opposite duality. Equation (2.16) does not lead to curvatures with definite duality, because the self-duality of $\Omega_{+}$guarantees the self-duality of the Riemann tensor $R_{\mu \nu}^{a b}\left(\Omega_{+}\right)$with respect to frame indices, but not necessarily with respect 
to coordinate indices. This is easily seen by making use of the modified exchange property of the Riemann curvature tensor

$$
R_{\mu \nu}^{a b}\left(\Omega_{+}\right)=R_{\mu \nu}^{a b}\left(\Omega_{-}\right)=e^{a \rho} e^{b \sigma} e_{c \mu} e_{d \nu} R_{\rho \sigma}^{c d}\left(\Omega_{-}\right)
$$

valid when the torsion tensor is closed, i.e. when $d H=0$. Since $\Omega_{-}$and, hence, $R\left(\Omega_{-}\right)$have no definite duality, the gauge field-strength resulting from the use of $(2.16)$

$$
F^{i}{ }_{\mu \nu}=\frac{1}{2} \eta^{i}{ }_{a b} R_{\mu \nu}{ }^{a b}\left(\Omega_{+}\right)
$$

is not self-dual. As for the other two possibilities, (2.14) and (2.15), they are both good starting points for a perturbative (with respect to $\alpha^{\prime}$ ) solution of the equations of motion. To enforce the vanishing of $\alpha^{\prime}$ corrections (at least up to second and third order) the condition $d H=0$ must be enforced. This requires the validity of the relation

$$
\operatorname{tr} R\left(\Omega_{-}\right) \wedge R\left(\Omega_{-}\right)=\frac{1}{30} \operatorname{Tr} F(A) \wedge F(A)
$$

which is not satisfied in general, unless one further restricts either the form of the metric $\widehat{g}_{\mu \nu}$ and/or the expression of the dilaton.

The first possibility has been considered in [6] and [4] with the conclusions that the non conformal part of the metric must be flat (in our notations this means $\widehat{g}_{\mu \nu}=\delta_{\mu \nu}$ ) and that, given the form of the supersymmetric ansatz (2.10) for $H$, the Bianchi identity, $d H=0$, requires the dilaton factor to obey the Laplace-Beltrami equation in flat background, i.e.

$$
e^{-2 \phi} \partial^{\mu} \partial_{\mu} e^{2 \phi}=0
$$

In this paper we would like to concentrate on the second option. The choice (2.14) is consistent with the identification of $F$ with $R\left(\Omega_{-}\right)$implied by (2.20), only if the dilaton is constant and the torsion is zero. In this case, the solution (2.10) is completely specified by the choice of a self-dual metric $\widehat{g}_{\mu \nu}$. 
In sect. 2.2 we will prove that the class of solutions found in [4] (corresponding to the choice (2.15)) are "dual" in the sense of [13] to the class of solutions of our interest here.

To make more explicit the ansatz (2.10) we will specify the form of the self-dual background metric, $\widehat{g}_{\mu \nu}$. Several classes of self-dual metrics are known in literature [18, 30]. We will concentrate on Asymptotically Locally Euclidean metrics. Our main motivation is that for generic (even not self-dual) ALE metrics the euclidean Einstein-Hilbert action is conjectured to be semipositive definite [31]. This is of course a necessary prerequisite for the sake of giving a mathematically sound definition of a quantum theory of gravity in terms of functional integrals.

Geometrical properties of four-dimensional ALE manifolds are well known in literature [18] and are recalled for completeness in Appendix B.

\subsection{World-Sheet Interpretation of heterotic ALE instantons}

In this section we would like to give a world-sheet interpretation to the propagation of the string in the heterotic ALE instanton backgrounds we have previously described, that is to say, we want to study the corresponding $\sigma$-models which describe the propagation of the string degrees of freedom associated to the four-dimensional Euclidean $x$-space.

Apart from the bosonic coordinates of the string, denoted by $X^{\mu}$, one has to consider their superpartners, $\Psi^{\mu}$, which from the two-dimensional point of view are left-handed fermions, and, in order to include the extra heterotic degrees of freedom, four additional right-handed fermions, which we denote by $\bar{\Lambda}^{a}$.

The action of the two-dimensional $\sigma$-model describing the propagation of the heterotic string in a background with metric $G_{\mu \nu}$, antisymmetric tensor $B_{\mu \nu}$ and gauge fields $A_{\mu}^{i}$ is given by $[2,3]$

$$
\begin{aligned}
& S=\frac{1}{4 \pi \alpha^{\prime}} \int d z d \bar{z}\left\{G_{\mu \nu}(X) \partial X^{\mu} \bar{\partial} X^{\nu}+B_{\mu \nu}(X) \partial X^{\mu} \bar{\partial} X^{\nu}\right. \\
& \left.+i G_{\mu \nu}(X) \Psi^{\mu} \bar{D}_{-} \Psi^{\nu}+i \delta_{a b} \bar{\Lambda}^{a} D_{+} \bar{\Lambda}^{b}+F_{\mu \nu}^{i}(A) \eta_{i a b} \Psi^{\mu} \Psi^{\nu} \bar{\Lambda}^{a} \bar{\Lambda}^{b}\right\}
\end{aligned}
$$


where the covariant derivatives are defined by $\left(\partial=\partial_{z}, \bar{\partial}=\partial_{\bar{z}}\right)$

$$
\begin{aligned}
& \bar{D}_{-} \Psi^{a}=\bar{\partial} \Psi^{a}+\Omega_{\nu-}^{a b} \bar{\partial} X^{\nu} \Psi_{b} \\
& D_{+} \bar{\Lambda}^{b}=\partial \Lambda^{a}+A^{i}{ }_{\nu} \eta_{i}{ }^{a b} \partial X^{\nu} \Lambda_{b}
\end{aligned}
$$

with $\Omega_{\nu-}^{a b}=\widehat{\omega}_{\nu}^{a b}-H_{\nu}^{a b}$ the spin connection with torsion. In (2.22) we have neglected the dilaton term [29], because it is of higher order in $\alpha^{\prime}$.

When the metric is hyperkählerian $[32,8]$, the $\sigma$-model $(2.22)$ is invariant under extended supersymmetries. In particular this is true, if one takes $G_{\mu \nu}$ to be an ALE metric and sets $B_{\mu \nu}$ to zero, as in the solution of our interest here. Besides the usual left-handed supersymmetry which on the bosonic coordinates $X^{\mu}$ induces the transformation

$$
\delta X^{\mu}=\epsilon^{(0)} \Psi^{\mu}
$$

(2.22) is invariant under three extra supersymmetries [8] whose action on the $X^{\mu}$ is

$$
\delta X^{\mu}=\epsilon^{(i)} J_{i}{ }^{\mu}{ }_{\nu} \Psi^{\nu} \quad i=1,2,3
$$

with the J's the three covariantly constant complex structures defined in (B.4). All of this is in agreement with the known result that non-linear $\sigma$-models on hyperkähler manifolds (a class of manifolds which includes four-dimensional ALE manifolds, see Appendix B) admit four left-handed supersymmetries. Actually, thanks to (2.14), (2.22) admits four right-handed extra supersymmetries which can be exposed by interpreting the fermions $\bar{\Lambda}^{a}$ as (frame components of) righthanded superpartners of the $X^{\mu}$. Indeed it can be shown that (2.22) is invariant also under the four additional supersymmetric transformations whose action on the $X^{\mu}$ is

$$
\delta X^{\mu}=\bar{\epsilon}^{(A)} \bar{J}_{A}{ }^{\mu}{ }_{\nu} E^{\nu}{ }_{a} \bar{\Lambda}^{a} \quad A=0,1,2,3
$$

where the $E^{\nu}{ }_{a}$ are the components of the $\sigma$-model tetrad and $\bar{J}_{0}{ }^{\mu}{ }_{\nu}=\delta^{\mu}{ }_{\nu}$. 
This proves that (2.22) with the standard embedding (2.14) and constant dilaton has $N=(4,4)$ supersymmetries on the world-sheet. An analogous observation was made in $[6,4]$ with the conclusion that lowest order solutions of the string equations of motion, built from the standard embedding (2.15), are exact solutions to all orders in $\alpha^{\prime}$, because of the expected absence of radiative corrections in the corresponding two-dimensional $N=(4,4) \sigma$-models.

In fact the authors of [6] could even go further and exhibit, in the limiting case of an asymptotically vanishing dilaton, an exact conformal field theory which describes the string propagation on a semi-wormhole geometry coupled to a 't Hooft instanton with winding number $\nu=1$. From now on we will call this solution the CHS solution.

The main difference between the heterotic ALE solutions we have described in sect. 2.1 and the CHS solution lies in the non triviality of the metric. Since the conformal factor of the $\sigma$-model metric of the CHS solution is the exponential of the dilaton field, the corresponding "canonical" metric is flat. In this case, even in the presence of a non vanishing axionic torsion, as it happens with the ansatz (2.10), no gravitino zero-modes are expected which could give rise to a non trivial gravitino condensate.

In the sense of Buscher's duality [13] ALE instantons or, more in general, selfdual multi-center metrics are tightly related to the multi-monopole solutions of [4]. Starting from the fact that the dilaton of the multi-monopole solution has exactly the form of the potential $V(\vec{x})$ in the Gibbons-Hawking metric (B.1) (see (B.3)), one can go from the self-dual multi-center metrics to the conformally flat multi-monopole solutions.

Duality with respect to both abelian and non-abelian isometries for self-dual metrics have already been considered [33]. Without going into details and referring directly to $[13,33]$, one can show that a $\sigma$-model such as $(2.22)$ can be related to

\footnotetext{
$\star$ For product spaces, like $\left(M_{4} \otimes M_{6}\right)$, with block diagonal metrics, as the ones we are considering here, the appropriate Weyl rescalings are given by (2.5) with $D=4$
} 
a dual $\sigma$-model by exploiting the existence of isometries of the initial metric. In the case of the multi-center metric (B.1) the relevant $U(1)$ isometry is generated by the Killing vector $\frac{\partial}{\partial \tau}$. The formulae for the transformation to the dual metric, dilaton and antisymmetric tensor fields are given by

$$
\begin{aligned}
G_{00}^{D} & =\frac{1}{G_{00}}, \quad G_{0 i}^{D}=\frac{B_{0 i}}{G_{00}}, \quad G_{i j}^{D}=G_{i j}-\frac{1}{G_{00}}\left(G_{0 i} B_{0 j}+G_{0 j} B_{0 i}\right) \\
B_{0 i}^{D} & =\frac{G_{0 i}}{G_{00}}, \quad B_{i j}^{D}=B_{i j}+\frac{1}{G_{00}}\left(G_{0 i} B_{0 j}-G_{0 j} B_{0 i}\right) \\
\Phi^{D} & =\Phi-\frac{1}{2} \log \left(G_{00}\right)
\end{aligned}
$$

where D stands for dual. The index 0 refers to the cyclic coordinate $\tau$, while the index $i$ is relative to the other three cartesian coordinates, $x^{i}$. Using the explicit expression of the metric (B.1), which is already expressed in adapted coordinates, one gets

$$
\begin{aligned}
G_{00}^{D} & =V(\vec{x}), \quad G_{0 i}^{D}=0, \quad G_{i j}^{D}=V(\vec{x}) \delta_{i j} \\
B_{0 i}^{D} & =\omega_{i}, \quad B_{i j}^{D}=0 \\
\Phi^{D} & =\Phi_{0}+\frac{1}{2} \log (V(\vec{x}))
\end{aligned}
$$

The gauge field $A_{\mu}^{D}$ is still obtained by using the standard embedding equation, whose validity is preserved by the duality transformation. As it is evident from (2.28), the dual metric is conformally flat with a conformal factor which exactly equals the exponential of the dilaton. Thus, as announced, the canonical metric turns out to be flat, all the non triviality of the solution being tranferred to the dilaton-axion system. It is nice to see that, thanks to the standard embedding and the peculiar form of multi-center metric following from (B.2), the dilaton-axion system satisfies the condition for a supersymmetric background, i.e.

$$
H_{\mu \nu \rho}^{D}=\sqrt{G^{D}} \varepsilon_{\mu \nu \rho}^{\sigma} \partial_{\sigma} \Phi^{D}
$$

and that the resulting gauge field-strength $F_{\mu \nu}^{D}$ is self-dual. 
For $\epsilon=1$ the dual backgrounds coincide with the multi-monopole solutions found in [4], which in this way appear to be dual to Euclidean multi-Taub-NUT spaces. Connections on Euclidean multi-Taub-NUT spaces are self-dual and admit a "standard embedding", similarly to ALE spaces [11]. Notice, however, that multiTaub-NUT spaces are asymptotically Euclidean only in three directions.

Even if the solutions we have discussed above are conjectured to be exact solutions to all orders in $\alpha^{\prime}$, it seems very difficult to find an exact "algebraic" conformal field theory which would describe the string propagation on these selfdual backgrounds (and their dual theories) at generic point of their moduli space. Further duality transformations with respect to non-abelian isometries [33] do not seem to give any clue for the solution of the problem.

In spite of these difficulties, $\sigma$-models with ALE instanton backgrounds in a particular singular limit, have been identified with solvable $\mathbb{C}^{2} / \Gamma$ orbifold conformal field theories, $\Gamma$ being a Kleinian subgroup of $S U(2)$ [34]. In order to recover the propagation of the string on a smooth manifold one must perturb the orbifold conformal field theory along the "truly marginal deformations" given by the moduli of the (orbifold) background. The authors of [34] have shown that there exists a precise correspondence between the "short representations" of the $N=4$ superconformal algebra and the moduli of metric and antisymmetric tensor, although they have not been able to determine the exact transformation connecting "geometric" and " $\sigma$-model" bases. Notice that models perturbed along marginal directions correspond to superconformal field theories with $c=(6,6)$ (which is the value of the conformal anomaly of four flat supercoordinates).

In the construction of a solution to the classical equations of motion of the $D=10$ heterotic (or type I) superstring theory, we have been, so far, rather cavalier about the dynamics and the geometry of the extra six dimensions, $\left\{y^{i} ; i=\right.$ $0,1, \cdots, 5\}$. On this question we essentially have two alternatives. One is to interpret our four-dimensional ALE solutions in terms of the propagation of a flat five-brane in a five-dimensional space-time, $y_{0}$ being the time coordinate. In this 
picture the complete ten-dimensional solution would correspond to an $N=(4,4)$ $\sigma$-model, whose action is the sum of a non trivial part (eq. (2.22)) plus a free part describing the dynamics of the six-dimensional $y$-coordinates. In this case the four-dimensional effective field theory will have $N=2$ space-time supersymmetry.

Alternatively, in view of a possible application to the study of dynamical SUSY breaking, it seems more appealing to interpret our heterotic ALE instantons as solutions of the Euclidean equations of motion of a $D=4, N=1$ effective supersymmetric theory. In this case the six-dimensional space is taken to be a Calabi-Yau threefold. The corresponding internal conformal field theory has $N=(2,2)$ supersymmetry. The spectrum of massless fields is consequently enlarged, but if we embed the istantonic $S U(2)$ group in the hidden $E(8)$, the ALE solution would still remain a solution of the equations of motion. In fact the ALE solution only involves space-time fields arising from the universal ("identity") sector of the internal superconformal field theory which is independent of the details of the compactification.

\subsection{A Particular Solution: the Heterotic Eguchi-Hanson instan-} TON

In the previous subsection we have found a general class of solutions of the effective low energy theory arising from the underlying heterotic string theory. We now specialize our solution to the Eguchi-Hanson gravitational instanton [17] which corresponds to a two center metric $(k=1)$ with $\epsilon=0$ and $m=\frac{1}{2}$ in (B.3). Placing the two centers, $\vec{x}_{1}, \vec{x}_{2}$, symmetrically along the $z$ axis, at a distance $\left|\vec{x}_{1}-\vec{x}_{2}\right|=\frac{a^{2}}{4}$, and performing the change of variables $(\vec{x} \equiv(x, y, z))[35]$

$$
\begin{array}{ll}
x=\frac{u^{2}}{8} \sin \theta \cos \psi & z=\frac{r^{2}}{8} \cos \theta \\
y=\frac{u^{2}}{8} \sin \theta \sin \psi & \tau=2 \varphi
\end{array}
$$


the line element (B.1) can be cast in the form

$$
d s^{2}=\widehat{g}_{\mu \nu} d x^{\mu} d x^{\nu}=\frac{d r^{2}}{1-\left(\frac{a}{r}\right)^{4}}+r^{2}\left(\sigma_{x}^{2}+\sigma_{y}^{2}+\left(1-\left(\frac{a}{r}\right)^{4}\right) \sigma_{z}^{2}\right)
$$

where the radial variable is constrained by $r \geq a$ and we have introduced the definition $u=r \sqrt{1-\left(\frac{a}{r}\right)^{4}}, 0 \leq u \leq \infty$. The $\sigma$ 's are the left-invariant one-forms of the $S U(2)$ group and are defined in Appendix A. The global topology of the EH manifold, $M_{E H}$, is that of $T^{*}\left(S^{2}\right)$ (the cotangent bundle over the Riemann two-sphere). The slices at fixed $r$ have the topology of a distorted sphere (at $r \approx a M_{E H} \approx \mathbb{R}^{2} \times S^{2}$, at $\left.r \approx \infty M_{E H} \approx \mathbb{R} \times S^{3} / Z_{2}\right)$ with points, antipodal with respect to the origin, identified. This identification is necessary to remove the (apparent) "bolt" singularity at $r=a$. The boundary at $\infty$ is a real projective space, i.e. $R P_{3}=S^{3} / Z_{2}$.

The group of isometries of $M_{E H}$ is the subgroup $S U(2)_{R} \otimes U(1)_{L}$ of the Euclidean Lorentz group, $O(4) \approx S U(2)_{R} \otimes S U(2)_{L}$. The generators of $S U(2)_{R} \otimes$ $U(1)_{L}$ are given in terms of the four linearly independent Killing vectors, $\bar{\xi}_{(i)}^{\mu}, i=$ $1,2,3$ and $\xi_{(3)}^{\mu}$ (see (A.15), (A.19)), by the formulae

$$
\begin{aligned}
& \bar{\xi}_{(1)}^{\mu} \partial_{\mu}=\left(\sin \varphi \frac{\partial}{\partial \theta}-\frac{\cos \varphi}{\sin \theta} \frac{\partial}{\partial \psi}+\frac{\cos \theta \cos \varphi}{\sin \theta} \frac{\partial}{\partial \varphi}\right) \\
& \bar{\xi}_{(2)}^{\mu} \partial_{\mu}=-\left(\cos \varphi \frac{\partial}{\partial \theta}+\frac{\sin \varphi}{\sin \theta} \frac{\partial}{\partial \psi}-\frac{\cos \theta \sin \varphi}{\sin \theta} \frac{\partial}{\partial \varphi}\right) \\
& \bar{\xi}_{(3)}^{\mu} \partial_{\mu}=\frac{\partial}{\partial \varphi}, \\
& \xi_{(3)}^{\mu} \partial_{\mu}=\frac{\partial}{\partial \psi}
\end{aligned}
$$

where $0 \leq \theta \leq \pi, 0 \leq \phi \leq 2 \pi, 0 \leq \psi \leq 2 \pi$. The restricted range of $\psi$ is related to the $Z_{2}$ identification of antipodal points mentioned above. Indeed referring to the relation (A.13) between cartesian and spherical coordinates, it is immediately seen that the identification of points having $\psi$ differing by (multiples of) $2 \pi$ implies the identification of the points of the manifold that have opposite coordinates. 
For completeness we also report the definition of the generators of the coset space $S U(2)_{L} / U(1)_{L}$

$$
\begin{aligned}
& \xi_{(1)}^{\mu} \partial_{\mu}=\left(\sin \psi \frac{\partial}{\partial \theta}-\frac{\cos \psi}{\sin \theta} \frac{\partial}{\partial \varphi}+\frac{\cos \theta \cos \psi}{\sin \theta} \frac{\partial}{\partial \psi}\right) \\
& \xi_{(2)}^{\mu} \partial_{\mu}=-\left(\cos \psi \frac{\partial}{\partial \theta}+\frac{\sin \psi}{\sin \theta} \frac{\partial}{\partial \varphi}-\frac{\cos \theta \sin \psi}{\sin \theta} \frac{\partial}{\partial \psi}\right)
\end{aligned}
$$

These operators correspond to global symmetries of the classical lagrangian, broken by the EH background.

The EH tetrad one-forms are

$$
e^{a} \equiv e_{\mu}^{a} d x^{\mu}=\left\{\frac{r}{u} d r, r \sigma_{x}, r \sigma_{y}, u \sigma_{z}\right\}
$$

The self-dual spin connection one-forms, $\omega^{a b} \equiv \omega_{\mu}^{a b} d x^{\mu}=\frac{1}{2} \epsilon^{a b}{ }_{c d} \omega^{c d}$, are related, thanks to (2.14), to the gauge connection one-forms, $A^{i} \equiv A_{\mu}^{i} d x^{\mu}(i=1,2,3$ is the index of the $S U(2)$ adjoint representation). Using (2.30), one explicitely finds

$$
\begin{aligned}
\omega^{10}=\omega^{23} & =\frac{1}{2} A^{1}=\frac{u}{r} \sigma_{x} \\
\omega^{20}=\omega^{31} & =\frac{1}{2} A^{2}=\frac{u}{r} \sigma_{y} \\
\omega^{30}=\omega^{12} & =\frac{1}{2} A^{3}=\frac{u u^{\prime}}{r} \sigma_{z}
\end{aligned}
$$

where the prime means derivative with respect to $r$. For later use, we notice the identity

$$
\frac{i}{2} A_{\mu}^{k} \sigma_{k}=\frac{1}{4} \omega_{\mu}^{a b} \sigma_{a b}
$$

which immediately follows from (2.14) and (A.4), i.e. from the fact that through (2.14) we are identifying the gauge connection, $A^{i}$, which belongs to the adjoint representation of the instantonic $S U(2)$ with the spin connection, $\omega^{a b}$, which similarly belongs to the adjoint representation of the $S U(2)_{L}$ subgroup of the Euclidean Lorentz group. 
We furthermore introduce the coefficient functions, $a^{(k)}$, defined by

$$
A_{\mu}^{k}=a^{(k)} e_{\mu}^{k} \quad k=1,2,3
$$

From (2.33) and (2.34) one explicitly gets

$$
a^{(1)}=a^{(2)}=\frac{2 u}{r^{2}} \quad a^{(3)}=\frac{2 u^{\prime}}{r}
$$

Finally we record the expressions of the curvature two-forms, $R^{a b} \equiv \frac{1}{2} R_{\mu \nu}^{a b} d x^{\mu} d x^{\nu}$ and $F^{i} \equiv \frac{1}{2} F_{\mu \nu}^{i} d x^{\mu} d x^{\nu}$

$$
\begin{aligned}
& R^{10}=R^{23}=\frac{1}{2} F^{1}=\frac{2}{r^{2}}\left(\frac{a}{r}\right)^{4}\left(e^{0} \wedge e^{1}-e^{2} \wedge e^{3}\right) \\
& R^{20}=R^{31}=\frac{1}{2} F^{2}=\frac{2}{r^{2}}\left(\frac{a}{r}\right)^{4}\left(e^{0} \wedge e^{2}-e^{3} \wedge e^{1}\right) \\
& R^{30}=R^{12}=\frac{1}{2} F^{3}=-\frac{4}{r^{2}}\left(\frac{a}{r}\right)^{4}\left(e^{0} \wedge e^{3}-e^{1} \wedge e^{2}\right)
\end{aligned}
$$

\section{Index Theorems for the Heterotic Eguchi-Hanson Instanton}

In this section we want to study the topological properties of the particular ALE solution, the heterotic EH instanton, described in sect. 2.3, and compute the indices of all relevant wave operators, using standard formulae from index theorems.

For the gravitational part all topological invariants have been computed in [18, 36]. The Euler characteristic, $\chi$, has both a "bulk" and a "boundary" contribution and it is given by the formula

$$
\chi=\frac{1}{32 \pi^{2}} \int_{M} \varepsilon^{a b c d}\left(R_{a b} \wedge R_{c d}\right)-\frac{1}{16 \pi^{2}} \int_{\partial M} \varepsilon^{a b c d}\left(\theta_{a b} \wedge R_{c d}-\frac{2}{3} \theta_{a b} \wedge \theta_{c e} \wedge \theta_{d}^{e}\right)
$$

with $\theta_{a b}$ the second fundamental forms of the Lorentz group i.e. the difference between the spin connection of the original metric, computed on the boundary, and the spin connection obtained from the boundary product-metric. 
If we take $M=M_{E H}{ }^{\star}$, the boundary, $\partial M$, will be represented by a slice at $r=r_{0}$, which in the end we will have to send to $\infty$. Since the EH metric factorizes on $\partial M$ one easily gets

$$
\begin{aligned}
& \theta_{01}=-\left(1-\left(\frac{a}{r_{0}}\right)^{4}\right)^{\frac{1}{2}} \sigma_{x} \\
& \theta_{02}=-\left(1-\left(\frac{a}{r_{0}}\right)^{4}\right)^{\frac{1}{2}} \sigma_{y} \\
& \theta_{03}=-\left(1+\left(\frac{a}{r_{0}}\right)^{4}\right) \sigma_{z} \\
& \theta_{12}=\theta_{23}=\theta_{31}=0
\end{aligned}
$$

It follows from (3.1) and (3.2)

$$
\chi_{E H}=\frac{3}{2}+\frac{1}{2}=2
$$

The Hirzebruch signature, $\tau$, receives contribution only from the "bulk" and it is given by

$$
\tau=-\frac{1}{24 \pi^{2}} \int_{M} \operatorname{tr}(R \wedge R)=b_{2}^{+}-b_{2}^{-}
$$

$\tau$ represents the difference between the number of normalizable antiself-dual and self-dual harmonic two-forms in the manifold. For the EH case one gets

$$
\tau_{E H}=-1
$$

The number of (left-handed) gravitino zero-modes, $\psi_{\mu}^{\alpha}$, i.e. the number of solutions of the equation

$$
\epsilon^{\lambda \mu \nu \rho} \bar{\sigma}_{\mu} D_{\nu} \psi_{\rho}=\epsilon^{\lambda \mu \nu \rho} \bar{\sigma}_{\mu}\left(\partial_{\nu}+\frac{1}{4} \omega_{\nu}^{a b} \sigma_{a b}\right) \psi_{\rho}=0
$$

turns out to be $2|\tau|=2$ in the $\mathrm{EH}$ case. The explicit expression of the two gravitino zero-modes will be presented in sect. 4 .

$\star$ From now on throughout this section the subscript $E H$ on $M$ will always be understood 
The Dirac equation for the gauge-singlet (left-handed) dilatino field, $\lambda^{\alpha}$,

$$
\not \phi_{S} \lambda \equiv \bar{\sigma}^{\mu} D_{\mu} \lambda \equiv \bar{\sigma}^{\mu}\left(\partial_{\mu}+\frac{1}{4} \omega_{\mu}^{a b} \sigma_{a b}\right) \lambda=0
$$

has no normalizable solutions. This result immediately follows from the observation that in the Ricci-flat EH background

$$
\left.\not D_{S} \not{ }_{S}\right|_{E H}=\left.\left(D^{2}+\frac{1}{8} R_{\mu \nu a b} \sigma^{\mu \nu} \sigma^{a b}\right)\right|_{E H}=\left.D^{2}\right|_{E H}
$$

if one remembers that in a non-compact background the equation $D^{2} \phi=0$ has no normalizable solutions.

Finally the expression of the Dirac operator acting on the (left-handed) gaugino, $\chi^{\alpha}$, is

$$
\not \supset \chi^{I}=\bar{\sigma}^{\mu}\left\{\left(\partial_{\mu}+\frac{1}{4} \omega_{\mu}^{a b} \sigma_{a b}\right) \delta_{K}^{I}+\frac{i}{2} f_{J K}^{I} A_{\mu}^{J}\right\} \chi^{K}=0
$$

where $f_{I J K}$ are the structure constants of the gauge group.

Since the istanton lives in an $S U(2)$ subgroup of the heterotic $E(8) \otimes E(8)$ (or $S O(32))$ group, it is necessary to decompose its adjoint representation in terms of representations of the istantonic $S U(2)$. As we have argued in sect. 2.2, it is convenient to embed the instantonic $S U(2)$ in the "hidden" $E(8)$. By decomposing this $E(8)$ with respect to its maximal subgroup, $S U(2) \otimes E(7)$, the 248-dimensional adjoint representation of $E(8)$ breaks as

$$
\underline{248}=(\underline{3}, \underline{1}) \oplus(\underline{2}, \underline{56}) \oplus(\underline{1}, \underline{133})
$$

We see that, for the maximal embedding we are considering here, only the fundamental and the adjoint representations of $S U(2)$ come into play. The Dirac equation for the singlet representation is identical to (3.7) and, as stated above, has no normalizable solutions. 
In order to find the number of zero-modes of the gaugino in the fundamental and in the adjoint representation of $S U(2)$ we recall the general formula for the index, ind $\left(\not D_{V}, M, \partial M\right)$, of the Dirac operator, $\not D_{V}$, coupled to the spin bundle over the manifold $M$ tensored with a vector bundle $V$. For our purposes, the YangMills connection on $V$ will be eventually identified with the spin connection. The formula for ind $\left(\not D_{V}, M, \partial M\right)$ reads

$$
\begin{aligned}
\operatorname{ind}\left(\not D_{V}, M, \partial M\right) & =\frac{\operatorname{dim} V}{192 \pi^{2}}\left[\int_{M} \operatorname{tr}(R \wedge R)-\int_{\partial M} \operatorname{tr}(\theta \wedge R)\right]-\frac{1}{8 \pi^{2}}\left[\int_{M} \operatorname{tr}_{V}(F \wedge F)\right. \\
& \left.-\int_{\partial M} \operatorname{tr}_{V}(\alpha \wedge F)\right]-\frac{1}{2}\left[\eta\left(\not D_{V}, \partial M\right)+h\left(\not D_{V}, \partial M\right)\right]
\end{aligned}
$$

In (3.10) there are three kinds of contributions. Contributions from the "bulk" which involve only the curvatures $R$ and $F$. Local boundary contributions, which besides $R$ and $F$ involve the corresponding second fundamental forms, $\theta$ and $\alpha$, on $\partial M$. Finally there are non-local boundary contributions given in terms of two invariants, $h\left(\not D_{V}, \partial M\right)$ and $\eta\left(\not D_{V}, \partial M\right)$.

The $\eta\left(\not D_{V}, \partial M\right)$-invariant is the analytic continuation to $s=0$ of the meromorphic function $\eta\left(\not D_{V}, \partial M, s\right)$ defined for $\Re(s)>2$ by

$$
\eta\left(\not D_{V}, \partial M, s\right)=\sum_{\lambda \neq 0}|\lambda|^{-s} \operatorname{sign} \lambda=\sum_{\lambda>0} \lambda^{-s}-\sum_{\lambda<0}(-\lambda)^{-s}
$$

with $\lambda$ 's the non-zero eigenvalue of $\left.\not \phi_{V}\right|_{\partial M}$. We remark that $\eta\left(\not D_{V}, \partial M\right)$ is left invariant by multiplication of the $\lambda$ 's by arbitrary positive real numbers and hence, in particular, by a conformal rescaling of the metric on $\partial M$.

The "harmonic correction" $h\left(\not D_{V}, \partial M\right)$ represents the number of zero eigenvalues of the Dirac operator on the boundary, $\left.\not D_{V}\right|_{\partial M}$ and coincides with the dimension of the space of the harmonic functions of the operator $D_{V}^{2}$ restricted to the boundary. 
Let's start the actual computation with the two "bulk" contributions. The first term in (3.10) is immediately evaluated using (3.4). The calculation of $\int_{M} \operatorname{tr}_{V}(F \wedge$ $F)$ is reduced to the previous one by observing that the embedding (2.14) implies

$$
2 \operatorname{tr}_{\underline{2}} F \wedge F=\operatorname{tr} R \wedge R
$$

and that for the $S U(2)$ generators in the representation $\underline{R}$ one has the identity

$$
\operatorname{tr}_{\underline{R}}\left(T^{a} T^{b}\right)=\frac{2}{3} t(t+1)(2 t+1) \operatorname{tr}_{\underline{2}}\left(T^{a} T^{b}\right)
$$

with $\operatorname{dim} \underline{R}=2 t+1$. Combining these results, one finds

$$
\operatorname{ind}\left(\not D_{V}, M, \partial M\right)_{b u l k}=3\left[\frac{2 t+1}{24}-\frac{1}{3} t(t+1)(2 t+1)\right]
$$

We will use this formula for $t=\frac{1}{2}, 1$ recalling (see (3.9)) that the adjoint representation of $E(8)$ contains one triplet and 56 doublets when decomposed with respect to the istantonic $S U(2)$.

As for the boundary contributions, the local terms vanish because by explicit computation the integrands go to zero, as $r_{0}$ go to $\infty$, faster than the integration measure. We now turn to the non-local boundary terms. As we shall see, the Dirac operator on the boundary has no zero eigenvalues implying

$$
h\left(\not D_{V}, \partial M\right)=0
$$

In order to compute the $\eta$-invariant, following [37], it is convenient to separate the Dirac operator on $\partial M$ into two terms, a gauge singlet part, in which only the singlet boundary Dirac operator, $\left.\not \phi_{S}\right|_{\partial M}$, appears and a gauge non-singlet part. The singlet Dirac operator on the boundary has the form

$$
-\left.i \not D_{S}\right|_{\partial M}=-i \tau^{p} e_{p}^{i}\left(\partial_{i}+\frac{1}{4} \omega_{i(\partial M)}^{p q} \tau_{p} \tau_{q}\right)
$$

where $i, p, q=1,2,3, a=0,1,2,3$ and the subscript $(\partial M)$ is to remind that the spin connection, $\omega$, is computed on $\partial M$, i.e. on a slice of constant $r=r_{0}$ (to be 
sent to $\infty$ at the end of the calculations). The appearance of the Pauli matrices, $\tau_{p}$, follows from the last equation in the formula (A.2). A semplification arises from the factorization property of the EH metric on the boundary, which imply the equations

$$
\omega_{(\partial M)^{p q}} \equiv \omega_{i(\partial M)}^{p q} d x^{i}=\left.\omega_{\mu(M)}^{p q} d x^{\mu}\right|_{\partial M}
$$

In fact together with the obvious vanishing of the components $\omega_{i(\partial M)}{ }^{0 p}$, the above formulae allow us to write

$$
-\left.\not \not D_{S}\right|_{\partial M}=-i \tau^{p} e_{p}^{i}\left(\partial_{i}+\left.\frac{1}{8} \omega_{i(M)}^{a b}\right|_{\partial M} \sigma_{a} \sigma_{b}\right)
$$

so that the expression of the Dirac operator on the boundary, $\partial M$, appears as that of a (three-dimensional) Dirac operator in which the spin connection part is effectively that of the manifold $M$, only restrited to $\partial M$, i.e. computed at $r=r_{0}$. To get the explicit expression of (3.18) we first rewrite it in the form

$$
-\left.i \not \phi_{S}\right|_{\partial M}=-i \tau^{p} e_{p}^{i}\left(\partial_{i}+\frac{1}{2}\left(\frac{i}{2} A_{i}^{k} \sigma_{k}\right)\right)
$$

having used (2.35). Then from the identity

$$
\frac{1}{4} \tau^{p} e_{p}^{i} A^{k} \sigma_{k}=\frac{1}{4} \sum_{i=1}^{3} a^{(i)}
$$

which follows from (2.35), and the explicit expressions of the coefficients, $a^{(i)}$, given in (2.37), (3.19) finally becomes

$$
-\left.\not \not D_{S}\right|_{\partial M}=2\left(\begin{array}{cc}
L_{3} & L_{-} \\
L_{+} & L_{3}
\end{array}\right)+\frac{3}{2}\left(\begin{array}{ll}
1 & 0 \\
0 & 1
\end{array}\right)
$$

where the differential operators $L_{ \pm}$and $L_{3}$ are defined in Appendix A. 
Using the previous notations, one finds, with a little algebra, for the boundary Dirac operator in the doublet representation

$$
-\left.i \not \underline{D}_{\underline{2}}\right|_{\partial M}=\left(\begin{array}{cc}
-i \not D_{S} & 0 \\
0 & -i \not D_{S}
\end{array}\right)+\left(\begin{array}{cc}
\tau_{3} & \tau_{1}-i \tau_{2} \\
\tau_{1}+i \tau_{2} & -\tau_{3}
\end{array}\right)
$$

To lighten the notation we have dropped the subscript $\partial M$ on $\not D_{S}$ inside the matrix in the r.h.s. of (3.22). The very form of $-\left.i \not \underline{D}_{\underline{2}}\right|_{\partial M}$ suggests that we solve the eigenvalue equation

$$
-\left.i \not \underline{2}_{\underline{2}}\right|_{\partial M} \Psi=\lambda \Psi
$$

by expanding $\Psi$ in terms of the $S U(2)$ rotation matrices, $D_{n, m}^{l}(\theta, \phi, \psi)$, whose definition is recalled in Appendix A. In this way the infinite dimensional eigenvalue problem for the differential operator $\left.\not_{\underline{2}}\right|_{\partial M}$ becomes a finite dimensional eigenvalue problem for a $4 \mathrm{x} 4$ matrix with non-vanishing determinant. This fact implies $\lambda \neq 0$, thus the validity of equation (3.15).

For generic, fixed, values of $l, n$ and $m$ (notice that the index $m$ is not acted upon by the operator (3.22)) there are four distinct eigenvectors that may be written in the form

$$
\Psi_{n, m}^{(i) l}=\left(\begin{array}{c}
c_{1}^{(i)} D_{n-1, m}^{l} \\
c_{2}^{(i)} D_{n, m}^{l} \\
c_{3}^{(i)} D_{n, m}^{l} \\
c_{4}^{(i)} D_{n+1, m}^{l}
\end{array}\right) \quad i=1,2,3,4
$$

with suitable coefficients, $c_{k}^{(i)}$, whose explicit expression we will not need here. Given the restrictions on the allowed values of $n$ and $m$ following from the definition of the $D_{n, m}^{l}$, the form (3.24) of the eigenvectors will retain its validity only if $l \neq 0$, $|m| \leq l$ and $|n| \leq l-1$, because otherwise some of the entries in (3.24) will loose their meaning. We will discuss separately these limiting cases below. 
The four eigenvalues corresponding to the four eigenvectors (3.24) are

$$
\begin{aligned}
\lambda_{n, m}^{(1) l} & =2 l+\frac{5}{2} & \lambda_{n, m}^{(3) l} & =-2 l+\frac{1}{2} \\
\lambda_{n, m}^{(2) l} & =2 l+\frac{1}{2} & \lambda_{n, m}^{(4) l} & =-2 l-\frac{3}{2}
\end{aligned}
$$

All of them have identical multiplicity, $d$, equal to the product of the degeneracies of the values of the two "magnetic" indices, allowed by the form (3.24) of the eigenvector $\Psi_{n, m}^{(i) l}$, i.e. $d=d_{m} d_{n}=(2 l+1)(2 l-1)$.

As we mentioned above, besides the eigenvectors and eigenvalues given by (3.24) and (3.25) there are few exceptional cases in which either $l=0$ or $n$ violates the restriction $|n| \leq l-1$. For $l \neq 0$ the proper form of the eigenvector is obtained by setting to zero in the expression (3.24) the entries which loose meaning when $n$ takes the limiting values $n= \pm l$ or $n= \pm(l+1)$. For $l=0$ all the entries in (3.24) should be taken as $\theta, \phi, \psi$-independent constants. In detail one has

(i) $n=l+1$

The last three entries in (3.25) have to be set to zero: 1 eigenvector, $\Psi_{l+1, m}^{l}$, with eigenvalue $2 l+\frac{5}{2}$ and multiplicity $d=2 l+1$;

(ii) $n=-l-1$

The first three entries in (3.25) have to be set to zero: 1 eigenvector, $\Psi_{-l-1, m}^{l}$, with eigenvalue $2 l+\frac{5}{2}$ and multiplicity $d=2 l+1$;

(iii) $n=l$

The last entry in (3.25) has to be set to zero: 3 eigenvectors, $\Psi_{l, m}^{(k) l}, k=1,2,3$, with eigenvalues $2 l+\frac{5}{2}, 2 l+\frac{1}{2},-2 l-\frac{3}{2}$, all with multiplicity $d=2 l+1$;

(iv) $n=-l$

The first entry in (3.25) has to be set to zero: 3 eigenvectors, $\Psi_{-l, m}^{(k) l}, k=$ $1,2,3$, with eigenvalues $2 l+\frac{5}{2}, 2 l+\frac{1}{2},-2 l-\frac{3}{2}$, all with multiplicity $d=2 l+1$;

(v) $l=0$

2 constant eigenvectors with eigenvalues $\frac{5}{2},-\frac{3}{2}$ and multiplicity $d=3,1$ respectively. 
We are now in position to compute $\eta\left(\not D_{\underline{2}}, \partial M\right)$ using formula (3.11). We first notice that the identification of antipodal points in the EH manifold forces the sum over the angular momentum, $l$, implicit in (3.11), to run only over integral values. The contribution from $l=0$ is easily evaluated and gives

$$
\eta\left(\not \phi_{\underline{2}}, \partial M, s\right)_{l=0}=\frac{3}{\left(\frac{5}{2}\right)^{s}}-\frac{1}{\left(\frac{3}{2}\right)^{s}}
$$

A little more work is necessary to compute the contribution from $l \neq 0$ which turns out to be

$$
\begin{aligned}
\eta\left(\not_{\underline{2}}, \partial M, s\right)_{l \neq 0} & =\frac{1}{2^{s}} \sum_{l=1}^{\infty}\left[\frac{(2 l+1)(2 l+3)}{\left(l+\frac{5}{4}\right)^{s}}+\frac{(2 l+1)^{2}}{\left(l+\frac{1}{4}\right)^{s}}\right. \\
& \left.-\frac{(2 l+1)^{2}}{\left(l+\frac{3}{4}\right)^{s}}-\frac{(2 l+1)(2 l-1)}{\left(l-\frac{1}{4}\right)^{s}}\right] \\
& =\frac{1}{2^{s}}\left[4 \zeta\left(s-2, \frac{9}{4}\right)-2 \zeta\left(s-1, \frac{9}{4}\right)-\frac{3}{4} \zeta\left(s, \frac{9}{4}\right)+\frac{3}{4} \zeta\left(s, \frac{3}{4}\right)\right. \\
& +4 \zeta\left(s-2, \frac{5}{4}\right)+2 \zeta\left(s-1, \frac{5}{4}\right)+\frac{1}{4} \zeta\left(s, \frac{5}{4}\right)-4 \zeta\left(s-2, \frac{7}{4}\right) \\
& \left.+2 \zeta\left(s-1, \frac{7}{4}\right)-\frac{1}{4} \zeta\left(s, \frac{7}{4}\right)-4 \zeta\left(s-2, \frac{3}{4}\right)-2 \zeta\left(s-1, \frac{3}{4}\right)\right]
\end{aligned}
$$

The sum of the various Riemann zeta functions in (3.27) is evaluated by using the known relation between the Riemann zeta functions and the Bernoulli polynomials, which reads $\zeta(-n, q)=-\frac{B_{n+1}(q)}{n+1}$. Adding (3.26) to (3.27) and taking the limit $s \rightarrow 0$, one gets for the $\eta$-invariant

$$
\eta\left(\not \phi_{\underline{2}}, \partial M\right)=\eta\left(\not D_{\underline{2}}, \partial M\right)_{l=0}+\eta\left(\not D_{\underline{2}}, \partial M\right)_{l \neq 0}=2-\frac{5}{2}=-\frac{1}{2}
$$

Inserting (3.14), (3.15) and (3.28) in (3.10), we finally find

$$
\operatorname{ind}\left(\not D_{\underline{2}}, M, \partial M\right)=-1
$$

The equality of $\operatorname{ind}\left(\not \not_{\underline{2}}, M, \partial M\right)$ with the value (3.5) of the Hirzebruch signature can be traced back to the observation that, for the doublet representation, isospin 
$\frac{1}{2}$ and spin $\frac{1}{2}$ can be combined together to get a total spin of 1 or 0 . We can then compute the index of the Dirac operator in the doublet representation by relating it to a formula (to be found in [18]) that gives the index of the generalized Dirac operator acting on "spinorial forms" of left-handed spin $\frac{m}{2}$ and right-handed spin $\frac{n}{2}$. These "spinorial forms" are sections of a spin bundle which is the symmetric tensor product of $m$ copies of the elementary spin bundle of a left-handed spinor and of $n$ copies of the elementary spin bundle of a right-handed spinor. In the EH case this formula reduces to

$$
\begin{aligned}
\operatorname{ind}\left(\not D_{\left(\frac{m}{2}, \frac{n}{2}\right)}\right) & =\frac{1}{32}(m+1)(n+1)\left[(-1)^{m}-(-1)^{n}\right] \\
& +\frac{(m+1)(n+1)}{240}\left[m(m+2)\left(3 m^{2}+6 m-14\right)\right. \\
& \left.-n(n+2)\left(3 n^{2}+6 n-14\right)\right] \tau
\end{aligned}
$$

A straightforward application of (3.30) leads indeed to the results ind $(\not D(1,0))=\tau$ and $\operatorname{ind}\left(\not D_{(0,0)}\right)=0$.

The above observation will be exploited in the next section, where we will find the explicit form of the (left-handed) doublet zero-mode. Since no righthanded zero-modes are expected in a self-dual background such as the heterotic EH instanton, the unique doublet zero-mode should be identified with the one obtainable from the (unique, $\left|\tau_{E H}\right|=1$ ) normalizable closed self-dual two-form existing on the EH manifold.

The calculation of $\operatorname{ind}\left(\not D_{\underline{3}}, M, \partial M\right)$ can be performed in complete analogy with the previous case. In order to separate the angular momentum dependence, instead of using the standard form of the generators in the adjoint representation, $\left(T^{i}\right)_{j k}=$ $-i\left(\varepsilon^{i}\right)_{j k}$, it turns out to be more convenient to diagonalize $T^{3}$ and write

$$
T^{3}=\left(\begin{array}{ccc}
1 & 0 & 0 \\
0 & 0 & 0 \\
0 & 0 & -1
\end{array}\right), T^{1}=\frac{1}{\sqrt{2}}\left(\begin{array}{ccc}
0 & -1 & 0 \\
-1 & 0 & 1 \\
0 & 1 & 0
\end{array}\right), T^{2}=\frac{1}{\sqrt{2}}\left(\begin{array}{ccc}
0 & i & 0 \\
-i & 0 & -i \\
0 & i & 0
\end{array}\right)
$$

The Dirac operator which will now act on a spinor of the form $\vec{\Psi}=\left(\Psi^{1}, \Psi^{2}, \Psi^{3}\right)$ 
with $\Psi^{k}, k=1,2,3$, two-component spinors, takes the form

$$
-\left.i \not \underline{\beta}_{\underline{3}}\right|_{\partial M}=\left(\begin{array}{ccc}
-i \not D_{S} & 0 & \\
0 & -i \not D_{S} & 0 \\
0 & 0 & -i \not \phi_{S}
\end{array}\right)+2\left(\begin{array}{ccc}
2 \tau_{3} & -\tau_{-} & 0 \\
-\tau_{+} & 0 & \tau_{-} \\
0 & \tau_{+} & -2 \tau_{3}
\end{array}\right)
$$

where $\tau_{+}=\sqrt{2}\left(\tau_{1}+i \tau_{2}\right), \tau_{-}=\sqrt{2}\left(\tau_{1}-i \tau_{2}\right)$.

For generic, fixed, values of $l, m$ and $n$, with $l \neq 0,|m| \leq l$ and $-l+1 \leq n \leq l-2$ (seven special cases will have to be considered separately), there are six distinct eigenvectors. They have the form

$$
\Psi_{n, m}^{(i) l}=\left(\begin{array}{c}
c_{1}^{(i)} D_{n-1, m}^{l} \\
c_{2}^{(i)} D_{n, m}^{l} \\
c_{3}^{(i)} D_{n, m}^{l} \\
c_{4}^{(i)} D_{n+1, m}^{l} \\
c_{5}^{(i)} D_{n+1, m}^{l} \\
c_{6}^{(i)} D_{n+2, m}^{l}
\end{array}\right) \quad i=1, \ldots, 6
$$

with suitable coefficients, $c_{k}^{(i)}$, whose explicit expression we will not need here. The corresponding six eigenvalues are

$$
\begin{aligned}
\lambda_{n, m}^{(1) l} & =2 l+\frac{7}{2} & \lambda_{n, m}^{(4) l} & =-2 l-\frac{5}{2} \\
\lambda_{n, m}^{(2) l} & =2 l+\frac{3}{2} & \lambda_{n, m}^{(5) l} & =-2 l-\frac{1}{2} \\
\lambda_{n, m}^{(3) l} & =2 l-\frac{1}{2} & \lambda_{n, m}^{(6) l} & =-2 l+\frac{3}{2}
\end{aligned}
$$

all with identical multiplicity, $d$, equal to the product of the degeneracies of the values of the two "magnetic" indices, allowed by the form (3.33) of the eigenvector $\Psi_{n, m}^{(i) l}$, i.e. $d=d_{m} d_{n}=(2 l+1)(2 l-2)$.

The seven special cases in which some of the entries of (3.33) loose meaning are separately listed below. 
(i) $n=l+1$

The last five entries in (3.33) have to be set to zero: 1 eigenvector, $\Psi_{l+1, m}^{l}$, with eigenvalue $2 l+\frac{7}{2}$ and multiplicity $d=2 l+1$;

(ii) $n=-l-2$

The first five entries in (3.33) have to be set to zero: 1 eigenvector, $\Psi_{-l-2, m}^{l}$, with eigenvalue $2 l+\frac{7}{2}$ and multiplicity $d=2 l+1$;

(iii) $n=l$

The last three enties in (3.33) have to be set to zero: 3 eigenvectors, $\Psi_{l, m}^{(k) l}, k=$ $1,2,3$, with eigenvalues $2 l+\frac{7}{2}, 2 l+\frac{3}{2},-2 l-\frac{5}{2}$, all with multiplicity $d=2 l+1$;

(iv) $n=-l-1$

The first three entries in (3.33) have to be set to zero: 3 eigenvectors, $\Psi_{-l-1, m}^{(k) l}, k=1,2,3$, with eigenvalues $2 l+\frac{7}{2}, 2 l+\frac{3}{2},-2 l-\frac{5}{2}$, all with multiplicity $d=2 l+1$;

(v) $n=l-1$

The last entry in (3.33) has to be set to zero: 5 eigenvectors, $\Psi_{l-1, m}^{(k) l}, k=$ $1, \ldots, 5$, with eigenvalues $2 l+\frac{7}{2}, 2 l+\frac{3}{2}, 2 l-\frac{1}{2},-2 l-\frac{1}{2},-2 l-\frac{5}{2}$, all with multiplicity $d=2 l+1$;

(vi) $n=-l$

The first entry in (3.33) has to be set to zero: 5 eigenvectors, $\Psi_{-l, m}^{(k) l}, k=$ $1, \ldots, 5$ with eigenvalues $2 l+\frac{7}{2}, 2 l+\frac{3}{2}, 2 l-\frac{1}{2},-2 l-\frac{1}{2},-2 l-\frac{5}{2}$, all with multiplicity $d=2 l+1$;

(vii) $l=0$

2 constant eigenvectors with eigenvalues $\frac{7}{2},-\frac{5}{2}$ and multiplicity $d=4,2$ respectively.

Putting all these results together, we get $\eta\left(D_{\underline{3}}, \partial M\right)=\frac{3}{4}$ and finally

$$
\operatorname{ind}\left(\not D_{\underline{3}}, M, \partial M\right)=\operatorname{ind}\left(\not D_{\underline{3}}, M, \partial M\right)_{b u l k}-\frac{3}{8}=-\frac{45}{8}-\frac{3}{8}=-6
$$

Exactly six left-handed zero-modes should be found for the triplet Dirac operator 
in the heterotic EH instanton, because no right-handed zero-modes are expected in a self-dual background. Their explicit expressions as well as those of all the bosonic and fermionic zero-modes will be given in the next section.

\section{Zero-modes and Their Geometrical Interpretation}

In this section we will present the explicit form of the zero-modes of the wave operators that control the quadratic fluctuations of the lagrangian (2.1), (2.2), expanded around the heterotic EH instantonic classical solution we have discussed in sect. 2.3.

These calculations are propaedeutical to any computational scheme which is intended to extend the instanton calculus, developed in globally supersymmetric theories, to the case of supergravity [26]. With an eye to future applications [27] to semi-classical instanton calculations, we will give the explicit expression of the fermionic and bosonic zero-modes in the the gauge-fixed form most appropriate for this purpose. The natural choice for the gauge-fixing of the functional integral $[19,20,23,27]$ induce the following gauge conditions on the gauge vector, graviton and gravitino zero-modes:

(i) background transversality for the gauge zero-modes;

(ii) background transversality and tracelessness for the graviton zero-modes;

(iii) background $\gamma$-tracelessness for the gravitino zero-modes.

As is well known, zero-modes of the bosonic fields are in one to one correspondence with the collective coordinates or moduli of the classical solution. They correspond to explicit parameters appearing in the instantonic solution or to classical symmetries which are broken by it. Zero-modes of the fermionic fields are associated to the superpartners of the bosonic collective coordinates. We present in the Table a summary of the results on zero-modes and collective coordinates which we are now going to derive in detail. 


\begin{tabular}{|c|c|c|c|c|}
\hline \multicolumn{2}{|r|}{ Field } & $n_{0}$ & Collective & Broken symmetry \\
\hline $\mathrm{B}_{\mu \nu}$ & axion & 1 & $b$ & Peccei-Quinn shift \\
\hline$\psi_{\mu}^{\alpha}$ & gravitino & 2 & $\eta^{\alpha}$ & Supersymmetry \\
\hline \multirow{2}{*}{$\mathrm{g}_{\mu \nu}$} & \multirow{2}{*}{ metric } & \multirow{2}{*}{3} & $a$ & Dilatations \\
\hline & & & $\beta_{1}, \beta_{2}$ & $\begin{array}{l}\mathrm{SU}(2)_{\mathrm{L}} / \mathrm{U}(1)_{\mathrm{L}} \\
\text { rotations }\end{array}$ \\
\hline$A_{\mu}^{i}$ & $\begin{array}{l}\text { gauge vector } \\
\text { (triplet) }\end{array}$ & 12 & $\gamma_{1}, \ldots, \gamma_{12}$ & $\begin{array}{c}\text { Global SU(2) gauge } \\
\text { otations = tangent bundle } \\
\text { deformations }\end{array}$ \\
\hline$\chi_{\alpha}^{i}$ & $\begin{array}{l}\text { gaugino } \\
\text { (triplet) }\end{array}$ & 6 & $\alpha_{1}, \ldots, \alpha_{6}$ & $\gamma$ superpartners \\
\hline$A_{\mu}^{r}$ & $\begin{array}{l}\text { gauge vectors } \\
\text { (doublets) }\end{array}$ & 112 & $\theta_{1}, \ldots, \theta_{112}$ & $\begin{array}{c}\text { Global } \mathrm{E}_{8} / \mathrm{SU}(2) \otimes \mathrm{E}_{7} \\
\text { gauge rotations }\end{array}$ \\
\hline$\chi_{\alpha}^{r}$ & $\begin{array}{l}\text { gaugino } \\
\text { (doublets) }\end{array}$ & 56 & $\delta_{1}, \ldots, \delta_{56}$ & $\theta$ superpartners \\
\hline
\end{tabular}

Zero-modes and collective coordinates for heterotic Eguchi-Hanson Instanton

Following [16, 23], the construction of the gravitino and graviton zero-modes can be easily carried out, by exploiting properties of the $\mathrm{EH}$ manifold that are listed below.

(i) In the EH manifold there exists a closed self-dual two-form whose explicit components, in the frame specified by the tetrad (2.33), are

$$
B_{a b}=\eta_{a b}^{3}\left(\frac{a}{r}\right)^{4}
$$

(ii) The self-duality of the spin connection and the vanishing of the curvature scalar of the EH metric imply that both the square of the Rarita-Schwinger 
operator and the Liechnerowicz operator (the latter governs the propagation of the fluctuations of the metric) are identical in the chosen background gauge to the Laplace-Beltrami operator acting on self-dual two-forms [36].

(iii) In any self-dual background there always exist two covariantly constant right-handed spinors, $\bar{\epsilon}_{\dot{\alpha}}^{(r)}, r=1,2$. Actually in the EH background they are just constant spinors.

Using properties (i) to (iii), we conclude that the spinor components of the two spin $\frac{3}{2}$ zero-modes should have the expression

$$
\psi_{\alpha \beta \dot{\alpha}}^{(r)}=B_{a b} \sigma_{\alpha \beta}^{a b} \bar{\epsilon}_{\dot{\alpha}}^{(r)}, \quad r=1,2
$$

These zero-modes can be rewritten as Rarita-Schwinger spinors in the form

$$
\psi_{\mu}^{(r)}=D_{\mu} \eta^{(r)}-\frac{1}{4} \sigma_{\mu} \not D \eta^{(r)}
$$

having introduced the left-handed spinor $\eta^{(r)}=u \eta_{0}^{(r)}$ with $\eta_{0}^{(r)}=i \sigma_{0} \bar{\epsilon}^{(r)}$. The second term in the r.h.s. of (4.3) has been added to insure the validity of the gauge condition $\gamma_{\mu} \psi^{\mu}=0$. It can be interpreted as a global super Weyl rescaling, since $\left(\not D \eta^{(r)}\right)_{\dot{\alpha}}=\bar{\epsilon}_{\dot{\alpha}}^{(r)}=$ const.

The above construction is telling us that the existence of two gravitino zeromodes should be ascribed to the lack of invariance of the EH background under the two global supersymmetry transformations induced by the two left-handed spinors, $\eta^{(r)}$.

The three independent spin 2 zero-modes can be similarly constructed by performing a further supersymmetry transformation on (4.3). One obtains

$$
h_{\alpha \beta \dot{\alpha} \dot{\beta}}^{(I)}=\psi_{\alpha \beta \dot{\alpha}}^{(r)} \bar{\epsilon}_{\dot{\beta}}^{(s)} C_{r s}^{(I)}, \quad I=0,1,2
$$

where we have defined the Clebsch-Gordan coefficients

$$
C_{r s}^{(0)}=\delta_{r s} \quad C_{r s}^{(1)}=\left(\tau_{3}\right)_{r s} \quad C_{r s}^{(2)}=\left(\tau_{1}\right)_{r s}
$$


In the more standard tensor notation we can write

$$
h_{\mu \nu}^{(I)}=\psi_{\mu}^{(r)} \sigma_{\nu} \bar{\epsilon}^{(s)} C_{r s}^{(I)}+\psi_{\nu}^{(r)} \sigma_{\mu} \bar{\epsilon}^{(s)} C_{r s}^{(I)}
$$

Substituting (4.3) in (4.6) and using again the fact that $\bar{\epsilon}$ is covariantly constant, the expression (4.6) can be cast in the form of an infinitesimal diffeomorphism accompanied by a global Weyl rescaling. One can in fact write

$$
\begin{aligned}
h_{\mu \nu}^{(I)} & =C_{r s}^{(I)} \eta_{0}^{(r)} \sigma_{a} \bar{\epsilon}^{(s)}\left(\nabla_{\mu}\left(u e_{\nu}^{a}\right)+\nabla_{\nu}\left(u e_{\mu}^{a}\right)-\frac{1}{2} \nabla \cdot\left(u e^{a}\right) g_{\mu \nu}\right) \\
& =\nabla_{\mu} \zeta_{\nu}^{(I)}+\nabla_{\nu} \zeta_{\mu}^{(I)}-\frac{1}{2}\left(\nabla \cdot \zeta^{(I)}\right) g_{\mu \nu}
\end{aligned}
$$

with

$$
\zeta_{\mu}^{(I)}=\eta^{(r)} \sigma_{\mu} \bar{\epsilon}^{(s)} C_{r s}^{(I)}
$$

In (4.7) the derivative $\nabla_{\mu}$ contains only the Christoffel connection, while $D_{\mu}$ is fully covariant. Actually the last term in (4.7) in non-zero only for $I=0$ because, as it can be easily shown, $\nabla \cdot \zeta^{(1,2)}=0$, while $\nabla \cdot \zeta^{(0)}=$ constant.

With a suitable interpretation of the infinitesimal diffeomorphisms $\zeta_{\mu}^{(I)}$ these zero-modes may be related to the lack of invariance of the EH metric under dilatations and under the two rotations in the coset $S U(2)_{L} / U(1)_{L}$ (the $U(1)_{L}$ quotient factor is present because the corresponding generator, $\xi_{(3)}$, given in $(2.31)$, is a Killing vector of the EH metric).

This can be explicitely seen by separating the diffeomorphisms in (4.7) into a "rigid" part, $\xi_{(I)}^{\mu}$, related to dilations $(I=0)$ or to the two rotations in the coset $S U(2)_{L} / U(1)_{L}(I=1,2)$, and a "gauge" transformation part, $\Lambda_{(I)}^{\mu}$, whose presence is necessary to ensure the transversality of the corresponding zero-modes, $h_{\mu \nu}^{(I)}$.

The infinitesimal diffeomorphism related to dilatations can be split as follows

$$
\zeta_{(0)}^{\mu}=\left(\frac{u}{r}\right)^{2} x^{\mu}=x^{\mu}-\left(\frac{a}{r}\right)^{4} x^{\mu} \equiv \xi_{(0)}^{\mu}+\Lambda_{(0)}^{\mu}
$$

where the first term, $\xi_{(0)}^{\mu}=x^{\mu}$, clearly corresponds to a global rescaling. Similarly the other two zero-modes are associated to the global rotations induced by the two 
vector fields

$$
\zeta_{(i)}^{\mu}=u e_{(i)}^{\mu}=\eta_{i}{ }^{\mu}{ }_{\nu} x^{\nu}+\left(\frac{u}{r}-1\right) \eta_{i}{ }_{\nu}{ }_{\nu} x^{\nu} \equiv \xi_{(i)}^{\mu}+\Lambda_{(i)}^{\mu}
$$

with $i=1,2$ and $\eta_{i}{ }^{\mu}{ }_{\nu}$ the 't Hooft symbols.

As is well known [35], the metric (2.30) is obtained from (B.1) with a change of variables which puts the (dipole with) endpoints $\vec{x}_{1}, \vec{x}_{2}$ along a preferred axis. The length of this dipole is related to the modulus $a$. The effect of the rotations $\xi_{(1,2)}^{\mu}$ is to change the alignment of the dipole and that of the dilatation $\xi_{(0)}^{\mu}$ to rescale the distance $\left|\vec{x}_{1}-\vec{x}_{2}\right|$.

A more suggestive form of the graviton zero-modes, $h_{\mu \nu}^{(I)}$, can be obtained by noticing that the "rigid" transformations considered above can be interpreted as Lie derivatives along the congruences of the vector fields $\zeta_{(I)}^{\mu} \partial_{\mu}$. The curves of the congruences are generated by the exponential maps of these vector fields and can be parametrized by the moduli of the Eguchi-Hanson instanton, $a$ for the dilatations and $\beta_{1}, \beta_{2}$ for the two rotations.

The Lie derivative represents a diffeomorphism along the congruence, i.e. an infinitesimal change of the moduli, and can thus be also seen as the derivative of the metric with respect to the moduli themselves.

From the explicit form of the EH metric we have the formulae

$$
\begin{gathered}
\frac{\partial g_{\mu \nu}}{\partial \beta_{i}}=\xi_{(i)}^{\lambda} \partial_{\lambda} g_{\mu \nu}+g_{\mu \lambda} \partial_{\nu} \xi_{(i)}^{\lambda}+g_{\nu \lambda} \partial_{\mu} \xi_{(i)}^{\lambda} \\
=\nabla_{\mu} \xi_{(i) \nu}+\nabla_{\nu} \xi_{(i) \mu}, \quad i=1,2 \\
\frac{\partial g_{\mu \nu}}{\partial a} \mid=\xi_{(0)}^{\lambda} \partial_{\lambda} g_{\mu \nu}+g_{\mu \lambda} \partial_{\nu} \xi_{(0)}^{\lambda}+g_{\nu \lambda} \partial_{\mu} \xi_{(0)}^{\lambda}-\frac{1}{2}\left(\nabla \cdot \xi_{(0)}\right) g_{\mu \nu} \\
=\nabla_{\mu} \xi_{(0) \nu}+\nabla_{\nu} \xi_{(0) \mu}-\frac{1}{2}\left(\nabla \cdot \xi_{(0)}\right) g_{\mu \nu}
\end{gathered}
$$

The $\mid$ in the l.h.s. of (4.12) is to remember that the derivative with respect to $a$ must be taken by keeping constant the determinant of the metric. This constraint 
is responsible for the appearance of the last extra term in the r.h.s. of (4.12). The condition that the determinant of the metric should remain a constant comes, in turn, from the requirement that the fluctuation of the metric should be traceless.

Using equations (4.11) and (4.12), one can finally write

$$
\begin{aligned}
h_{\mu \nu}^{(0)} & =\frac{\partial g_{\mu \nu}}{\partial a} \mid+\nabla_{\mu} \Lambda_{\nu}^{(0)}+\nabla_{\nu} \Lambda_{\mu}^{(0)} \\
h_{\mu \nu}^{(1)} & =\frac{\partial g_{\mu \nu}}{\partial \beta_{1}}+\nabla_{\mu} \Lambda_{\nu}^{(1)}+\nabla_{\nu} \Lambda_{\mu}^{(1)} \\
h_{\mu \nu}^{(2)} & =\frac{\partial g_{\mu \nu}}{\partial \beta_{2}}+\nabla_{\mu} \Lambda_{\nu}^{(2)}+\nabla_{\nu} \Lambda_{\mu}^{(2)}
\end{aligned}
$$

In this way each graviton zero-mode is expressed as a sum of two terms. The first is the derivative of the $\mathrm{EH}$ metric with respect to a collective coordinate, the second is a gauge transformation necessary to make the zero-mode to obey the same gauge condition as the metric, i.e. to make it transverse (tracelessness is automatic from (4.7)).

We now turn to the study of the gaugino zero-modes, starting with the doublet case (for other gravitational instantons, analogous computations can be found in [38]). Using (2.35), we can write the Dirac equation in the doublet representation in the form

$$
-i \not D_{\underline{2}} \chi=-i \sigma^{a}{ }_{\alpha \dot{\alpha}} \partial_{a} \chi^{\alpha r}+\frac{i}{2} a^{(k)}\left(\delta_{\dot{\alpha} \beta} \delta^{r}{ }_{s}+\left(\tau^{k}\right)^{r}{ }_{s}\left(\tau^{k}\right)_{\dot{\alpha} \beta}\right) \chi^{\beta s}=0
$$

with $a^{(k)}$ defined in (2.37). It is easy to see that an ansatz of the form

$$
\chi^{\alpha r}=\left(\begin{array}{cc}
P_{1} D_{n-1, m}^{l} & Q_{1} D_{n, m}^{l} \\
P_{2} D_{n, m}^{l} & Q_{2} D_{n+1, m}^{l}
\end{array}\right)
$$

with $P_{1}, P_{2}, Q_{1}, Q_{2}$ purely radial functions ${ }^{\star}$ and $D_{n, m}^{l}$ the $S U(2)$ rotation matrices, leads to the following system of first order differential equations

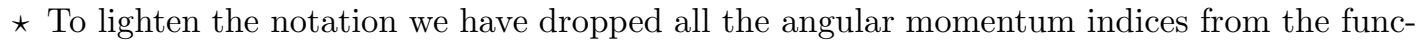
tions $P_{1}, P_{2}, Q_{1}, Q_{2}$
} 


$$
\begin{aligned}
{\left[\frac{u}{r} \frac{d}{d r}+\frac{2(n+1)}{u}\right] P_{1}+\frac{2}{r} a_{n l} P_{2} } & =0 \\
{\left[\frac{u}{r} \frac{d}{d r}+\frac{2 u}{r^{2}}-\frac{2 n}{u}\right] P_{2}+\frac{2}{r} a_{n l} P_{1}+\frac{2 u}{r^{2}} Q_{1} } & =0 \\
{\left[\frac{u}{r} \frac{d}{d r}+\frac{2 u}{r^{2}}+\frac{2 n}{u}\right] Q_{1}+\frac{2}{r} b_{n l} Q_{2}+\frac{2 u}{r^{2}} P_{2} } & =0 \\
{\left[\frac{u}{r} \frac{d}{d r}-\frac{2(n-1)}{u}\right] Q_{2}+\frac{2}{r} b_{n l} Q_{1} } & =0
\end{aligned}
$$

where $a_{n l}=\sqrt{(l+n)(l-n+1)}, b_{n l}=\sqrt{(l-n)(l+n+1)}$.

To solve the system (4.16) we recall that the result of the index theorem (3.29) was $\operatorname{ind}\left(\not \not_{\underline{2}}, M, \partial M\right)=-1$, leading us to the conclusion that in the doublet case there is just one (left-handed) gaugino zero-mode, since no right-handed zero-modes are expected in a self-dual background. We are thus led to take $l=0$ in the ansatz (4.15). With this choice the matrix (4.15) becomes off-diagonal. Since furthermore $a_{00}=b_{00}=0$, the system (4.16) is immediately solved, giving

$$
\chi^{\alpha r}=\left(\frac{a}{r}\right)^{4}\left(\begin{array}{ll}
0 & 1 \\
1 & 0
\end{array}\right)^{\alpha r}
$$

This formula is very much reminiscent of the form of the harmonic two-form (4.1). In fact there is another, more geometrical, way to determine the solution (4.17), which we now want to explain. Combining isospin and spin indices, one can separate the symmetric and the antisymmetric part of $\chi$ by writing

$$
\chi^{\alpha r}=U \varepsilon^{\alpha r}+\left(V_{a b} \sigma^{a b}\right)^{\alpha r}
$$

where $V_{a b}$ is self-dual. The Dirac equation becomes

$$
\bar{\sigma}^{\mu}\left[\varepsilon D_{\mu} U+\sigma^{a b} D_{\mu} V_{a b}\right]=0
$$

and thanks to the completeness of the $\bar{\sigma}^{\mu}$ matrices, one gets

$$
d U+* d V=0
$$

Acting with $d$ or $* d *$ allows to derive from (4.20) two separate equations. The 
first one, $d U=0$, has the only solution $U=$ constant, which is not normalizable. The second one, $* d V=0$ or, equivalently, $d V=0$ (since $V_{a b}$ is self-dual) implies that $V$ is a closed self-dual two-form. As there exists only one (normalizable) self-dual two-form on the EH manifold, $V_{a b}$ must coincide with $B_{a b}$ of equation (4.1). This procedure is very general and can be used to find the zero-modes of the Dirac operator in the doublet representation of the gauge group, in any self-dual background, whenever spin and gauge connections are identified.

We now discuss the Dirac equation for the triplet. Due to the lack of a clear geometrical interpretation, this case will turn out to be much more involved than the previous one.

The triplet Dirac operator has the form

$$
-i \not \underline{D}_{\underline{3}}=\left(\begin{array}{ccc}
-i \not D_{S} & 0 & 0 \\
0 & -i \not D_{S} & 0 \\
0 & 0 & -i \not D_{S}
\end{array}\right)+\left(\begin{array}{ccc}
B \tau_{3} & -A \tau_{-} & 0 \\
-A \tau_{+} & 0 & A \tau_{-} \\
0 & A \tau_{+} & -B \tau_{3}
\end{array}\right)
$$

where $A=a^{(1)}=a^{(2)}, B=a^{(3)}$. With an eye to the discussion of the eigenvalue problem for the boundary triplet Dirac operator in sect. 3, we look for zero-modes of the form

$$
\chi^{\alpha i}=\left(R^{1} D_{n-1, m}^{l}, R^{2} D_{n, m}^{l}, S^{1} D_{n, m}^{l}, S_{2} D_{n+1, m}^{l}, T^{1} D_{n+1, m}^{l}, T^{2} D_{n+2, m}^{l}\right)
$$

with $R^{1}, R^{2}, S^{1}, S^{2}, T^{1}, T^{2}$ purely radial functions ${ }^{\star}$ and $D_{n, m}^{l}$ the $S U(2)$ rotation matrices.

\footnotetext{
$\star$ To lighten the notation we have dropped all angular momentum indices from the functions $R^{1}, R^{2}, S^{1}, S^{2}, T^{1}, T^{2}$
} 
With the ansatz (4.22) the Dirac equation, $-i \not \underline{D}_{\underline{3}} \chi=0$, becomes

$$
\begin{aligned}
\left(D+\frac{2}{u} L_{3}\right) R^{1}+\frac{2}{r} L_{-} R^{2}+B R^{1} & =0 \\
\left(D-\frac{2}{u} L_{3}\right) R^{2}+\frac{2}{r} L_{+} R^{1}-B R^{2}-\sqrt{2} A S^{1} & =0 \\
\left(D+\frac{2}{u} L_{3}\right) S^{1}+\frac{2}{r} L_{-} S^{2}-\sqrt{2} A R^{2} & =0 \\
\left(D-\frac{2}{u} L_{3}\right) S^{2}+\frac{2}{r} L_{+} S^{1}+\sqrt{2} A T^{1} & =0 \\
\left(D+\frac{2}{u} L_{3}\right) T^{1}+\frac{2}{r} L_{-} T^{2}-B T^{1}+\sqrt{2} A S^{2} & =0 \\
\left(D-\frac{2}{u} L_{3}\right) T^{2}+\frac{2}{r} L_{+} T^{1}+B T^{2} & =0
\end{aligned}
$$

where we have introduced the definition

$$
D=\frac{u}{r} \frac{d}{d r}+\frac{2}{u}+\frac{u}{r^{2}}
$$

This is a linear system of six Fuchsian differential equations, equivalent to a Fuchsian differential equations of the sixth order, with three regular singular points for each one of the radial functions appearing in the ansatz (4.22). For special forms of the coefficients, a solution of a differential equation of the above type can be expressed in terms of the generalized hypergeometric series. This turns out not to be our case. We are thus forced to solve (4.23) by brute force, by trying the lowest possible values of the quantum numbers $l, n, m$, in the hope of successively reducing the number of independent equations in the system. We start with the choice $l=n=m=0$. In this case the equations decouple and one can easily find a solution which, however, turns out to be non-normalizable. Next we examine the case $l=1$ with $-3 \leq n \leq 2,-1 \leq m \leq 1$. The values of $n$ we have given are the only ones compatible with the assignment of angular momentum quantum numbers made in (4.22). The six resulting possible cases are pairwise symmetric and give rise to the following three situations.

(i) $n=-3,2$

The solution has only one non-zero component and it is not normalizable. 
(ii) $n=-2,1$

The solution has three non-zero components and it is not normalizable.

(iii) $n=-1,0$

The solution has five non-zero components and for each value of $n$ there are three (in correspondence with the three possible values of $m$ ) linearly independent normalizable solutions.

In order to explicitely find these solutions it is convenient to rewrite the system (4.23) as a fifth order differential equation by solving for one of the five non-zero components of (4.22). Fortunately three solutions of the resulting Fuchsian equation may be found with the ansatz: $x^{\alpha}(x-1)^{\beta}$, where $x=\left(\frac{a}{r}\right)^{4}$ and $\alpha, \beta$ are the exponents governing the behaviour of the solution around the singularities. Knowing three solutions it is possible to reduce the original fifth order differential equation to a second order differential equation of the known hypergeometric type and find the last two independent solutions. For each $n$ and $m$ one can construct five independent solutions of (4.23) and it just happens that only one linear combination of them is normalizable. Explicitely the six normalizable solutions are $(n=-1,0 ; m=-1,0,1)$

$$
\chi_{m}^{(\downarrow)}=\left(\begin{array}{c}
0 \\
-x^{\frac{3}{4}}(1+2 \sqrt{x}) D^{1}{ }_{-1, m} \\
x^{\frac{5}{4}} D^{1}-1, m \\
-\frac{1}{\sqrt{2}} x^{\frac{3}{4}} \sqrt{1-x} D_{0, m}^{1} \\
-\frac{x^{\frac{5}{4}} \sqrt{2(1-x)}}{1+\sqrt{x}} D_{0, m}^{1} \\
\frac{x^{\frac{3}{4}}(1-\sqrt{x})}{(1+\sqrt{x})} D^{1}{ }_{1, m}
\end{array}\right) \quad \chi_{m}^{(\uparrow)}=\left(\begin{array}{c}
-\frac{x^{\frac{3}{4}}(1-\sqrt{x})}{(1+\sqrt{x})} D^{1}{ }_{1, m} \\
\frac{x^{\frac{5}{4}} \sqrt{2(1-x)}}{1+\sqrt{x}} D^{1}{ }_{0, m} \\
-\frac{1}{\sqrt{2}} x^{\frac{3}{4}} \sqrt{1-x} D_{0, m}^{1} \\
x^{\frac{5}{4}} D^{1}{ }_{-1, m} \\
x^{\frac{3}{4}}(1+2 \sqrt{x}) D^{1}{ }_{-1, m} \\
0
\end{array}\right)
$$

This result is in agreement with the value of the index of the Dirac operator computed at the end of sect. 3 .

We conclude this section with a discussion on the relation between the number of triplet zero-modes and the number of moduli of an $S U(2)$ gauge instanton over 
the EH manifold. First of all notice that the number of zero-mode fluctuations of the gauge field is twice the (absolute) value of the index (3.35) for the triplet Dirac operator. This is a direct consequence of the already mentioned existence in self-dual backgrounds, such as the heterotic EH instanton, of two unbroken righthanded supersymmetries. Thanks to them, two zero-modes of the gauge field, $\delta A_{\mu}^{i(r)}$, can be generated out of each fermion zero-mode, $\chi_{\alpha}^{i}$, by writing

$$
\delta A_{\mu}^{i(r)}=\bar{\epsilon}_{\dot{\alpha}}^{(r)} \bar{\sigma}_{\mu}^{\dot{\alpha} \alpha} \chi_{\alpha}^{i}
$$

Thus the six zero-modes of the gaugino in the adjoint representation of the istantonic $S U(2)$ generate twelve zero-modes for quadratic operator of the gauge field fluctuactions around the hetrotic EH background. At this point the issue is to find a geometric interpretation for the corresponding 12 "collective coordinates" of the instantonic gauge connection. Following [39] and the discussion in Appendix $\mathrm{B}^{\star}$, we are led to identify these collective coordinates with the deformations of the tangent bundle in the adjoint representation of the structure group of the bundle, which in this case is $S L(2, \mathbb{C})$ (i.e. the complexification of $S U(2)$ ). The relevant cohomology group is $H^{1}(E n d T)$, consisting in the holomorphic $(0,1)$-forms with values in the adjoint representation of $S L(2, \mathbb{C})$ modulo gauge transformations. If our previous arguments are correct, we expect the dimension of the cohomology group $H^{1}(E n d T)$ to be 12. For the $\mathrm{EH}$ instanton this computation is not too difficult. Recalling that the EH manifold is the smooth resolution of the algebraic variety defined by the locus $W(x, y, z)=x y-z^{2}=0$ in $\mathbb{C}^{3}$, we see that the tangent bundle is an $S L(2, \mathbb{C})$ complex bundle whose sections (vector fields), $\vec{T}=\left(T^{x}, T^{y}, T^{z}\right)$, satisfy the condition

$$
y T^{x}+x T^{y}+2 z T^{z}=0
$$

We now want to deform the tangent bundlle into a new complex bundle, defined

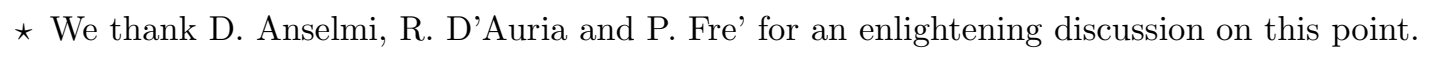


by

$$
P_{x} T^{x}+P_{y} T^{y}+P_{z} T^{z}=0
$$

but in such a way so as to mantain the vanishing of the first Chern class. The polynomial deformations of the tangent bundle $P_{x}, P_{y}, P_{z}$, which do not modify the first Chern class, can be identified as the polynomials in the variables $x, y, z$ of degree less or equal to the degree of the components of the gradient to the locus. In the case at hand we have $\vec{\nabla} W=(y, x, 2 z)$, so we need only to consider polynomials of degree one in $x, y, z$. Since each polynomial of degree one in three variables is identified by four coefficients, we conclude that the cohomology group $H^{1}(E n d T)$ has dimension $3=12$. At the moment we are not able to relate the algebro-geometric basis to the "physical" basis, given by the gauge field zero-modes (4.26). A proper understanding of this issue could help in determining the moduli space of instanton connections on the EH manifold.

\section{Conclusions and Perpectives}

In this paper we have shown that an arbitrary conformally self-dual gravitational background is solution of the classical equations of motion of the effective supergravity theory arising from the heterotic string. Specializing the gravitational background to the EH instanton, we have computed all the relevant fermionic and bosonic zero-modes.

The interest of all these calculations, which we have hinted in the Introduction, is in the fact that gravitational instantons may lead to SUSY breaking via the formation of gravitino and gaugino condensates.

It is one of the miracles of supersymmetry that the computation of suitable correlation functions, expanded around the saddle-point given by the classical instantonic solution, leads to finite and space-time independent results. It turns out that the computation of the functional integral gets reduced to the computation of a suitable combination of the zero-modes of the theory integrated over the 
moduli space of the instanton, since the determinants which are obtained from the functional integration over non-zero-modes cancel by supersymmetry. The first step in these calculations is thus the knowledge of the zero-modes themselves, which is the subject we hope to have settled in this work. This computations were first performed in [21] for global SUSY theories and in [23] for minimal $N=1$ supergravity. The present work should be the first of a series of papers in which instanton calculus is hopefully extended to generic supergravity theories, arising in the low energy limit of the heterotic string theory.

The deep reason why some correlation functions are constant in certain theories, probably lies in their connection with topological theories [15]. From a phenomenological point of view, theories with $N=1$ SUSY seem to be preferable with respect to those with $N=2^{\star}$, but in the former case, unfortunately, it has not been yet possible to relate the existence of constant correlation functions to known geometrical properties of the theory. Expecially in the case in which gravity is present such an interpretation would be highly desirable, given also the difficulty of defining in such theories, meaningful gauge invariant quantities. In a paper companion of this one we will discuss these matters together with the form of the Ward identities appropriate for $N=1$ supergravity.

Acknowledgements: We would like to acknowledge fruitful discussions with L. Alvarez-Gaumé, D. Anselmi, R. D’Auria, S. Ferrara, G. Gibbons, L. Girardello, C. Hull, K. Konishi, A. Sagnotti, M. Testa, and G. Veneziano. Two of us (M.B. and G.C.R.) would like to thank the organizers of the 1992 Triangular Meeting at Crete, where their collaboration started.

\footnotetext{
$\star$ A lot of progress has been recently made in the understanding of global $N=2$ supersymmetric theories[40]
} 


\section{APPENDIX A}

In this Appendix we establish the conventions and notations used throughout the paper. Indices from the beginning of the latin alphabet $(a, b, c, \cdots)$ are frame indices which range from 0 to 3 and are raised and lowered by the flat Euclidean metric. Indices from the middle of the latin alphabet $(i, j, k, \cdots)$ range from 1 to 3. Spinor indices are usually denoted by letters from the beginning of the greek alphabet $(\alpha, \beta, \gamma, \cdots)$. Indices from the middle of the greek alphabet $(\lambda, \mu, \nu, \cdots)$ denote coordinate indices which are raised and lowered by the metric tensor.

We fix the orientation of the volume measure by taking the permutation 1230 as the fundamental one and defining $\varepsilon^{1230}=+1$.

For the Euclidean Dirac matrices we use a Weyl basis

$$
\gamma^{a}=\left(\begin{array}{cc}
0 & \sigma_{\alpha \dot{\alpha}}^{a} \\
\bar{\sigma}_{a} \dot{\beta} \beta & 0
\end{array}\right)
$$

where:

$$
\begin{array}{ll}
\sigma_{\alpha \dot{\alpha}}^{0}=-i \delta_{\alpha \dot{\alpha}} & \sigma_{\alpha \dot{\alpha}}^{k}=\tau_{\alpha \dot{\alpha}}^{k} \\
\bar{\sigma}_{0}{ }^{\dot{\alpha} \alpha}=+i \delta^{\dot{\alpha} \alpha} & \bar{\sigma}_{k}{ }^{\dot{\alpha} \alpha}=\tau_{k}{ }^{\dot{\alpha} \alpha}
\end{array}
$$

with $\tau^{k} \equiv \tau_{k}$ the standard Pauli matrices.

Since the $\sigma^{a}$ 's form a complete set, one has

$$
\sigma^{a}{ }_{\alpha \dot{\alpha}} \bar{\sigma}_{a}{ }^{\dot{\beta} \beta}=2 \delta_{\alpha}{ }^{\beta} \delta_{\dot{\alpha}}^{\dot{\beta}}
$$

As usual we introduce the matrices

$$
\begin{aligned}
\sigma_{a b} & =\frac{1}{2}\left(\sigma_{a} \bar{\sigma}_{b}-\sigma_{b} \bar{\sigma}_{a}\right) \\
\bar{\sigma}_{a b} & =\frac{1}{2}\left(\bar{\sigma}_{a} \sigma_{b}-\bar{\sigma}_{b} \sigma_{a}\right)
\end{aligned}
$$


which satisfy the relations

$$
\begin{aligned}
& \sigma^{a}{ }_{\alpha \dot{\alpha}} \bar{\sigma}_{b}{ }^{\dot{\alpha} \beta}=\delta^{a}{ }_{b} \delta_{\alpha}{ }^{\beta}+\left(\sigma^{a}{ }_{b}\right)_{\alpha}{ }^{\beta} \\
& \bar{\sigma}_{a}{ }^{\dot{\alpha} \alpha} \sigma_{\alpha \dot{\beta}}^{b}=\delta_{a}^{b} \delta_{\dot{\beta}}^{\dot{\alpha}}+\left(\bar{\sigma}_{a}^{b}\right)_{\dot{\beta}}^{\dot{\alpha}}
\end{aligned}
$$

The matrices (A.4) are the generators of the Lorentz group $S U(2)_{L} \otimes S U(2)_{R}$ and can be also rewritten as

$$
\begin{aligned}
& \left(\sigma^{a}{ }_{b}\right)_{\alpha}{ }^{\beta}=+i\left(\eta_{k}\right)^{a}{ }_{b}\left(\tau^{k}\right)_{\alpha}{ }^{\beta} \\
& \left(\bar{\sigma}_{b}^{a}{ }_{b}\right)_{\dot{\beta}}^{\dot{\alpha}}=+i\left(\bar{\eta}_{k}\right)^{a}{ }_{b}\left(\tau^{k}\right)^{\dot{\alpha}}{ }_{\dot{\beta}}
\end{aligned}
$$

where $\left(\bar{\eta}_{k}{ }^{a b}\right) \eta_{k}^{a b}$ are the (anti)self-dual 't Hooft symbols

$$
\begin{aligned}
& \eta_{k}{ }^{a b}=\delta^{a}{ }_{k} \delta^{b}{ }_{0}-\delta^{a}{ }_{0} \delta^{b}{ }_{k}+\varepsilon_{k}{ }^{i j} \delta^{a}{ }_{i} \delta^{b}{ }_{j} \\
& \bar{\eta}_{k}{ }^{a b}=-\delta^{a}{ }_{k} \delta^{b}{ }_{0}+\delta^{a}{ }_{0} \delta^{b}{ }_{k}+\varepsilon_{k}{ }^{i j} \delta^{a}{ }_{i} \delta^{b}{ }_{j}
\end{aligned}
$$

They satisfy the relations

$$
\begin{aligned}
& \eta_{i}^{a b} \eta_{j b c}=-\delta_{i j} \delta^{a}{ }_{c}-\varepsilon_{i j}{ }^{k}\left(\eta_{k}\right)^{a}{ }_{c} \\
& \bar{\eta}_{i}{ }^{a b} \bar{\eta}_{j b c}=-\delta_{i j} \delta^{a}{ }_{c}-\varepsilon_{i j}{ }^{k}\left(\bar{\eta}_{k}\right)^{a}{ }_{c} \\
& \eta_{i}{ }^{a b} \eta^{i c d}=\delta^{a c} \delta^{b d}-\delta^{a d} \delta^{b c}+\varepsilon^{a b c d} \\
& \bar{\eta}_{i}{ }^{a b} \bar{\eta}^{i c d}=\delta^{a c} \delta^{b d}-\delta^{a d} \delta^{b c}-\varepsilon^{a b c d}
\end{aligned}
$$

The duality properties of the 't Hooft symbols induce the following duality properties of the Lorentz generators

$$
\begin{aligned}
\sigma^{a b} & =\frac{1}{2} \varepsilon^{a b}{ }_{c d} \sigma^{c d} \\
\bar{\sigma}^{a b} & =-\frac{1}{2} \varepsilon^{a b}{ }_{c d} \bar{\sigma}^{c d}
\end{aligned}
$$

Spinor indices are raised and lowered by the symplectic metric 


$$
\begin{array}{ll}
\varepsilon^{\alpha \beta}=i\left(\tau_{2}\right)^{\alpha \beta} & \varepsilon_{\alpha \beta}=-i\left(\tau_{2}\right)_{\alpha \beta} \\
\varepsilon^{\dot{\alpha} \dot{\beta}}=i\left(\tau_{2}\right)^{\dot{\alpha} \dot{\beta}} & \varepsilon_{\dot{\alpha} \dot{\beta}}=-i\left(\tau_{2}\right)_{\dot{\alpha} \dot{\beta}}
\end{array}
$$

which satisfy

$$
\begin{aligned}
& \varepsilon^{\alpha \beta} \varepsilon_{\beta \gamma}=\delta_{\gamma}^{\alpha} \\
& \varepsilon^{\dot{\alpha} \dot{\beta}} \varepsilon_{\dot{\beta} \dot{\gamma}}=\delta^{\dot{\alpha}} \dot{\gamma}
\end{aligned}
$$

As a consequence, the matrices $\bar{\sigma}^{a}$ and $\sigma^{a}$ are related by

$$
\bar{\sigma}_{a}^{\dot{\alpha} \beta}=-\varepsilon^{\alpha \beta} \sigma_{\beta \dot{\beta}}^{a} \varepsilon^{\dot{\alpha} \dot{\beta}}
$$

and the Lorentz generators with two upper indices are symmetric under the interchange of the spinor indices, i.e.

$$
\begin{aligned}
& \varepsilon^{\alpha \beta}\left(\sigma_{a b}\right)_{\beta}{ }^{\gamma}=\left(\sigma_{a b}\right)^{\alpha \gamma}=\left(\sigma_{a b}\right)^{\gamma \alpha} \\
& \varepsilon_{\dot{\alpha} \dot{\beta}}\left(\bar{\sigma}_{a b}\right)^{\dot{\beta}} \dot{\gamma}=\left(\bar{\sigma}_{a b}\right)_{\dot{\alpha}} \dot{\gamma}=\left(\bar{\sigma}_{a b}\right)_{\dot{\gamma}}^{\dot{\alpha}}
\end{aligned}
$$

In terms of the $\gamma$ matrices the duality properties of the Lorentz generators translate into the equations

$$
\begin{aligned}
\gamma^{a b c d} & =\varepsilon^{a b c d} \gamma_{5} \\
\gamma^{a b c} & =\varepsilon^{a b c d} \gamma_{5} \gamma_{d} \\
\gamma^{a b} & =-\frac{1}{2} \varepsilon^{a b c d} \gamma_{5} \gamma_{c d} \\
\gamma^{a} & =-\frac{1}{6} \varepsilon^{a b c d} \gamma_{5} \gamma_{b c d} \\
\mathbb{1} & =\frac{1}{24} \varepsilon^{a b c d} \gamma_{5} \gamma_{a b c d}
\end{aligned}
$$

Right-handed (dotted) spinors, $\epsilon$, satisfy the equation $\gamma_{5} \epsilon=-\epsilon$. Left-handed (undotted) spinors, $\eta$, satisfy the equation $\gamma_{5} \eta=\eta$. 
Using the 't Hooft symbols, one can define the left-invariant one-forms

$$
\sigma_{i}=\frac{1}{r^{2}} \eta_{i \mu \nu} x^{\mu} d x^{\nu}
$$

and the two-forms

$$
d \sigma_{i}=\frac{1}{r^{2}} \eta_{i \mu \nu} d x^{\mu} d x^{\nu}-\frac{2}{r^{4}} \eta_{i \mu \nu} x_{\rho} x^{\mu} d x^{\rho} d x^{\nu}
$$

where $r^{2}=x^{2}+y^{2}+z^{2}+t^{2}$. From (A.14) and (A.15) one has $d \sigma_{i}=\varepsilon_{i}{ }^{j k} \sigma_{j} \wedge \sigma_{k}$.

In terms of the spherical coordinates

$$
\begin{aligned}
& x+i y=r \cos \frac{\theta}{2} \exp \frac{i}{2}(\psi+\varphi) \\
& z+i t=r \sin \frac{\theta}{2} \exp \frac{i}{2}(\psi-\varphi)
\end{aligned}
$$

the left-invariant one-forms, $\sigma_{i}$ have the explicit expression

$$
\begin{aligned}
\sigma_{x} & =\frac{1}{2}(\sin \psi d \theta-\cos \psi \sin \theta d \varphi) \\
\sigma_{y} & =-\frac{1}{2}(\cos \psi d \theta+\sin \psi \sin \theta d \varphi) \\
\sigma_{z} & =\frac{1}{2}(d \psi+\cos \theta d \varphi)
\end{aligned}
$$

Their dual vector fields are (see (2.31), (2.32))

$$
L_{i}=-\frac{i}{2} \eta_{i \mu}{ }^{\nu} x^{\mu} \partial_{\nu} \equiv-i \xi_{(i)}^{\mu} \partial_{\mu}
$$

They satisfy the $S U(2)$ algebra: $\left[L_{i}, L_{j}\right]=i \varepsilon_{i j}{ }^{k} L_{k}$. In terms of spherical coordinates they read

$$
\begin{aligned}
& L_{1}=-i\left(\sin \psi \frac{\partial}{\partial \theta}-\frac{\cos \psi}{\sin \theta} \frac{\partial}{\partial \varphi}+\frac{\cos \theta \cos \psi}{\sin \theta} \frac{\partial}{\partial \psi}\right) \\
& L_{2}=i\left(\cos \psi \frac{\partial}{\partial \theta}+\frac{\sin \psi}{\sin \theta} \frac{\partial}{\partial \varphi}-\frac{\cos \theta \sin \psi}{\sin \theta} \frac{\partial}{\partial \psi}\right) \\
& L_{3}=-i \frac{\partial}{\partial \psi}
\end{aligned}
$$


Frequently used combinations of the first two vectors are

$$
L_{ \pm}=L_{1} \pm i L_{2}=e^{ \pm i \psi}\left(\mp \frac{\partial}{\partial \theta}+\frac{i}{\sin \theta} \frac{\partial}{\partial \varphi}-i \frac{\cos \theta}{\sin \theta} \frac{\partial}{\partial \psi}\right)
$$

In an analogous way one can define the right-invariant one-forms:

$$
\bar{\sigma}_{i}=\frac{1}{r^{2}} \bar{\eta}_{i \mu \nu} x^{\mu} d x^{\nu}
$$

or in terms of Euler angles:

$$
\begin{aligned}
& \bar{\sigma}_{x}=\frac{1}{2}(\sin \varphi d \theta-\cos \varphi \sin \theta d \psi) \\
& \bar{\sigma}_{y}=-\frac{1}{2}(\cos \varphi d \theta+\sin \varphi \sin \theta d \psi) \\
& \bar{\sigma}_{z}=\frac{1}{2}(d \varphi+\cos \theta d \psi)
\end{aligned}
$$

Their dual vector fields are (see (2.31))

$$
\bar{L}_{i}=-\frac{i}{2} \bar{\eta}_{i}^{\mu \nu} x_{\mu} \partial_{\nu} \equiv-i \bar{\xi}_{(i)}^{\mu} \partial_{\mu}
$$

They satisfy the $S U(2)$ algebra: $\left[\bar{L}_{i}, \bar{L}_{j}\right]=-i \varepsilon_{i j}^{k} \bar{L}_{k}$. In terms of spherical coordinates they read

$$
\begin{aligned}
& \bar{L}_{1}=-i\left(\sin \varphi \frac{\partial}{\partial \theta}-\frac{\cos \varphi}{\sin \theta} \frac{\partial}{\partial \psi}+\frac{\cos \theta \cos \varphi}{\sin \theta} \frac{\partial}{\partial \varphi}\right) \\
& \bar{L}_{2}=i\left(\cos \varphi \frac{\partial}{\partial \theta}+\frac{\sin \varphi}{\sin \theta} \frac{\partial}{\partial \psi}-\frac{\cos \theta \sin \varphi}{\sin \theta} \frac{\partial}{\partial \varphi}\right) \\
& \bar{L}_{3}=-i \frac{\partial}{\partial \varphi}
\end{aligned}
$$

The raising and lowering operators become

$$
\bar{L}_{ \pm}=\bar{L}_{1} \pm i \bar{L}_{2}=e^{ \pm i \varphi}\left(\mp \frac{\partial}{\partial \theta}+\frac{i}{\sin \theta} \frac{\partial}{\partial \psi}-i \frac{\cos \theta}{\sin \theta} \frac{\partial}{\partial \varphi}\right)
$$

The elements of the group $S U(2)$ may be parametrize in terms of the rotations 
matrices

$$
D_{R}(\theta, \varphi, \psi)=e^{i \psi T_{3}} e^{i \theta T_{2}} e^{i \varphi T_{3}}
$$

where the T's are the $S U(2)$ generators in the representation $\underline{R}$. The matrix elements of the rotation matrices

$$
D_{n, m}^{l}(\theta, \varphi, \psi)=e^{i n \psi} e^{i m \varphi} d_{n, m}^{l}(\theta)=\langle\theta, \phi, \psi \mid l, m, n\rangle \quad|m| \leq l,|n| \leq l
$$

yield a spin $l$ representations of the two $S U(2)$ groups defined above and one has

$$
\begin{aligned}
& L^{2}|l, m, n\rangle=l(l+1)|l, m, n\rangle \\
& L_{3}|l, m, n\rangle=n|l, m, n\rangle \\
& \bar{L}^{2}|l, m, n\rangle=l(l+1)|l, m, n\rangle \\
& \bar{L}_{3}|l, m, n\rangle=m|l, m, n\rangle
\end{aligned}
$$

The explicit expression of the matrices $d_{n, m}^{l}(\theta)$ are not needed in this work except for the case $l=1$, for which we have

$$
d_{n, m}^{1}(\theta)=\left(\begin{array}{ccc}
\frac{1+\cos \theta}{2} & \frac{\sin \theta}{\sqrt{2}} & \frac{1-\cos \theta}{2} \\
-\frac{\sin \theta}{\sqrt{2}} & \cos \theta & \frac{\sin \theta}{\sqrt{2}} \\
\frac{1-\cos \theta}{2} & -\frac{\sin \theta}{\sqrt{2}} & \frac{1+\cos \theta}{2}
\end{array}\right)
$$

The matrix elements of the raising and lowering operators can be derived from the equations

$$
\begin{aligned}
& L_{ \pm}|l, m, n\rangle=\sqrt{(l \mp n)(l \pm n+1)}|l, m, n \pm 1\rangle \\
& \bar{L}_{ \pm}|l, m, n\rangle=\sqrt{(l \mp m)(l \pm m+1)}|l, m \pm 1, n\rangle
\end{aligned}
$$

More details on rotation matrices and spinor weighted spherical harmonics can be found in [41] or in standard textbooks of Quantum Mechanics. 


\section{APPENDIX B}

In this Appendix we recall some known facts about the geometry of ALE manifolds with a special emphasis on those aspects that are more relevant for the problems we are dealing with in this paper.

The nice thing about ALE spaces is that they admit a complete topological classification, the so-called $A-D-E$ classification, in terms of the kleinian subgroups of $S U(2)$ [42]. Indeed to each one of such discrete subgroups, $\Gamma$, one can associate an ALE manifold, $M$, which is the smooth resolution of a certain singular variety in $\mathbb{C}^{3}$ with boundary $\partial M=S^{3} / \Gamma$. Referring to the existing literature on the subject and denoting by $\chi$ the Euler characteristic and by $\tau$ the Hirzebruch signature, let's recall the relevant facts about the $A-D-E$-classification. The classification is made in terms of two discrete series corresponding to the Dinkin diagrams, $A_{k}$ and $D_{k}$, and of the three exceptional cases corresponding to the exceptional groups $E_{6}, E_{7}$ and $E_{8}$. The $k^{t h}$ representative, $M_{A_{k}}$, of the $A$-series corresponds to the smooth resolution of the singular algebraic variety defined by the equation $x y=z^{k+1}$ in $\mathbb{C}^{3}$. Its boundary is the lens space $L(k+1,1)$ which coincides with $S^{3}$ modded out by the cyclic group $Z_{k+1}$, i.e. $\partial M_{A_{k}}=S^{3} / Z_{k+1}$. The manifold $M_{A_{k}}$ has $\chi=k+1, \tau=-k$. The $k^{\text {th }}$ representative, $M_{D_{k}}$, of the $D$-series corresponds to the resolution of the singular algebraic variety defined by the equation $x^{2} z+y^{2}=z^{k-1}$ in $\mathbb{C}^{3}$. Its boundary is $S^{3} / D_{k}^{*}$ with $D_{k}^{*}$ the double-covering of the dihedral group of order $k$. The manifold $M_{D_{k}}$ has $\chi=k+1, \tau=-k$. The three ALE spaces associated to the Dinkin diagrams of the exceptional groups can be described as follows. The $E_{6}$ representative, $M_{E_{6}}$, corresponds to the resolution of the variety $z^{4}=x^{2}+y^{3}$, its boundary is $S^{3} / T^{*}$ with $T^{*}$ the binary tetrahedral group. $M_{E_{6}}$ has $\chi=7, \tau=-6$. The $E_{7}$ representative, $M_{E_{7}}$, corresponds to the resolution of the variety $y z^{3}=x^{2}+y^{3}$, its boundary is $S^{3} / O^{*}$ with $O^{*}$ the binary octahedral group. $M_{E_{7}}$ has $\chi=8, \tau=-7$. The $E_{8}$ representative, $M_{E_{8}}$, corre-

sponds to the resolution of the variety $z^{5}=x^{2}+y^{3}$, its boundary is $S^{3} / I^{*}$ with $I^{*}$ the binary icosahedral group. $M_{E_{8}}$ has $\chi=9, \tau=-8$. Barring geometrical 
subtleties, once the singularity has been smoothed out, one can introduce on the resulting ALE manifold a Riemannian structure, i.e. a metric.

An explicit class of self-dual metrics, which corresponds to the ALE manifolds of the $A$-series, is given by the Gibbons-Hawking multi-center ansatz

$$
d s^{2}=V^{-1}(\vec{x})(d \tau+\vec{\omega} \cdot d \vec{x})^{2}+V(\vec{x}) d \vec{x} \cdot d \vec{x}
$$

with $V$ and $\vec{\omega}$ related by

$$
\vec{\nabla} V=\vec{\nabla} \times \vec{\omega}
$$

to ensure the self-duality of the curvature. Since the rigt-hand-side of eq.(B.2) is formally divergenceless up to point-like sources, the most general form of $V$ is

$$
V(\vec{x})=\epsilon+2 m \sum_{i=1}^{k+1} \frac{1}{\left|\vec{x}-\vec{x}_{i}\right|}
$$

with $\epsilon, m$ arbitrary parameters. Following the standard notations used in this context, in the above formulae we have set the Newton constant, $G_{N}$, equal to 1 . The choice of a common value of $m$ for all the sources is dictated by the requirement that the apparent Dirac-string singularities induced in $\omega$ be removable coordinate singularities of the metric. The choice $\epsilon=0, m=\frac{1}{2}$ corresponds to an admissible metric on the $k^{t h}$ representative of the $A$-series space, $M_{A_{k}}$. In particular the choice $k=0$ corresponds to a reparametrization of flat four-dimensional Euclidean space, while $k=1$ corresponds to the Eguchi-Hanson istanton which is discussed at length in this paper. The choice $\epsilon=1$ corresponds to the multi-Taub-NUT spaces which are neither complex nor ALE spaces.

From the observation that ALE metrics are conjectured to make the EinsteinHilbert action non-negative definite, we conclude that self-dual ALE metrics are absolute minima of the action, since in each set of topologically equivalent metrics, characterized by a common behavior at the boundary, self-dual metrics have zero action. 
For the sake of making contact with the complex geometry of supersymmetric backgrounds which may allow consistent propagation of the string, it is important to observe that ALE manifolds may be thought of as non compact Calabi-Yau manifolds $[43,34]$. Indeed, thanks to the self-duality of the curvature, the holonomy group of the ALE manifold with boundary $S^{3} / \Gamma$ is an $S U(2) / \Gamma$ subgroup of the Euclidean Lorentz group, $S O(4) \sim S U(2)_{L} \otimes S U(2)_{R}$. As a consequence in these backgrounds there always exist two covariantly constant right-handed spinors, $\bar{\epsilon}_{\dot{\alpha}}^{(r)}, r=1,2$, out of which one can construct three covariantly constant complex structures

$$
J_{a b}^{i}=\bar{\epsilon}^{(r)} \bar{\sigma}_{a b} \bar{\epsilon}^{(s)} \sigma_{r s}^{i}
$$

which together with the identity, $J_{a b}^{0}=\delta_{a b}$, satisfy the quaternionic algebra. Choosing one of the complex structures, say $J^{3}$, as the reference one, it is possible to introduce complex coordinates on the manifold and associate to it a closed twoform of type $(1,1), K=J_{a b}^{3} e^{a}{ }_{\mu} e^{b}{ }_{\nu} d x^{\mu} d x^{\nu}=K_{I \bar{J}} d z^{I} \wedge d z^{\bar{J}}$, which will play the role of Kähler form. This means that there exists a complex coordinate system in which the hermitean metric is kählerian. Actually ALE manifolds are hyperkählerian, due to the presence of the two other covariantly constant complex structures, $J^{1}$ and $J^{2}$. The latter generate (anti)holomorphic two-forms of type $(2,0)$ (or $(0,2)$ ) which may be interpreted as the nowhere vanishing (anti)holomorphic forms which characterize a Calabi-Yau manifold of complex dimension two. Since the only compact manifold of this class is known to be K3, one is led to identify ALE manifolds as "non-compact Calabi-Yau twofolds".

The local polynomial ring of the algebraic equation which characterizes an ALE manifold is the ring of deformations of its complex structure [42]. The number of generators of the ring for the $A$-series turns out to be $k$ and coincides with the number of complex parameters in the admissible deformations of the complex structure. These parameters could be reabsorbed through non-analytic changes of coordinates, in much the same way as one can eliminate the explicit dependence on the moduli from the metric of a Riemann surface. Another set of $k$ (real) 
parameters corresponds to the admissible deformations of the Kähler structure. In the case of the multi-center ansatz (B.1)-(B.3), once the origin of the threedimensional coordinate system has been chosen to coincide with one of the centers in the potential $V$, the metric will depend on $3 k$ parameters which can be made to coincide with the $3 k$ coordinates of the remaining centers. These $3 k$ parameters are in one to one correspondence with the zero-modes of the metric, i.e. with the zero-modes of the Liechnerowicz operator in the background given by the metric (B.1) with the choice $\epsilon=0, m=\frac{1}{2}$ in (B.3). The relevant cohomology group is $H^{(1,1)}\left(M_{A_{k}}, \partial M_{A_{k}}\right)$, i.e. the group of closed two-forms of Hodge type $(1,1)$ which vanish sufficiently fast on the boundary so as to have a finite norm. For ALE manifolds this cohomology group consists only of (harmonic) self-dual two-forms and has dimension $h_{(1,1)}=k$. Combining these $k$ self-dual two-forms with the three covariantly constant (and as such not normalizable) antiself-dual two-forms which are always present on hyperkähler manifolds, one gets $3 h_{(1,1)}=3 k$ deformations of the metric which can be shown to be zero-modes of the Liechnerowicz operator. Notice that for complex two-dimensional manifolds the relative cohomology group $H^{(1,1)}\left(M_{A_{k}}, \partial M_{A_{k}}\right)$ is responsible both for the complex deformations of the complex structure and for the real deformations of the Kähler class. The $k$ deformations of the Kähler structure can be "complexified" by taking as imaginary parts the $k$ self-dual two-forms, of type $(1,1)$. These two-forms can be interpreted as zero-mode fluctuations of the antisymmetric tensor around the corresponding ALE background.

The last topic we would like to briefly discuss in this Appendix is the deformation of the (vacuum) gauge bundle $[1,39]$. Through the "standard embedding" the gauge bundle is identified with the tangent bundle to the ALE manifold. Since for a Kähler manifold the curvature two-forms have only non-vanishing components of type $(1,1)$, the transition functions may be chosen to be holomorphic. The tangent bundle, $T_{M}$ or more simply $T$, turns out to be a holomorphic vector bundle. Thanks to the vanishing of the Ricci scalar, $T$ has vanishing first Chern class. Writing the algebraic equation of the singular variety in $\mathbb{C}^{3}$ associated to the 
ALE manifold in the implicit form, $W(x, y, z)=0$, the tangent vectors $\vec{t}$, satisfy

$$
t^{x} \frac{\partial W}{\partial x}+t^{y} \frac{\partial W}{\partial y}+t^{z} \frac{\partial W}{\partial z}=0
$$

In much the same way as an algebraic singularity can be deformed to a "nearby" (non-singular) variety through the elements of its polynomial ring, the defining equation of the tangent bundle (B.5) can be modified to a "nearby" equation with polynomial coefficients defining a new vector bundle $V$. The new holomorphic vector bundle $V$ may be equivalent to $T$ in the topological sense, but in general it will not be equivalent to $T$ in the holomorphic sense. In order to shift the initial solution of the heterotic string equations of motion to a "nearby" solution, admissible gauge bundle $V$ are naturally related to holomorphic deformations of $T$ which preserve the rank and the vanishing of the first Chern class. Somehow in analogy with the deformations of tangent bundles to hypersurfaces embedded in complex projective spaces $[1,39]$, the relevant deformations of (B.5) should be in one to one correspondence with the polynomials in $x, y, z$ of degree less or equal to the smallest among the degrees of $\frac{\partial W}{\partial x}, \frac{\partial W}{\partial y}$ and $\frac{\partial W}{\partial z}$. In view of the identification between the gauge connection and the connection on the tangent bundle, these deformations should correspond to zero-mode fluctuations of the gauge field around the considered background. In two complex dimensions selfduality and holomorphicity are tightly bound. In complex coordinates $z^{I}, I=1,2$ a hermitean self-dual connection, $A$, has components:

$$
\begin{aligned}
& A_{I}=U^{-1} \partial_{I} U \\
& A_{\bar{I}}=\left(\partial_{\bar{I}} \bar{U}\right) \bar{U}^{-1}
\end{aligned}
$$

with $U \neq \bar{U}$. The connection (B.6) is not a pure gauge. The components of Hodge type $(2,0)$ and $(0,2)$ of the field-strength $F$ vanish, but the components of type $(1,1)$ do not. Deforming the tangent bundle to a new vector bundle with selfdual curvature (field-strength) is tantamount to find a new gauge connection such that the above properties of $F$ are mantained. This means that the zero-modes 
of the gauge connection are in one to one correspondence with holomorphic oneforms in the adjoint representation of the structure group. The structure group is $S L(2, \mathbb{C})$, which is the complexification of the $S U(2)$ group where the gauge istanton lives. The relevant deformations are those belonging to the cohomology group $H^{1}(\operatorname{End}(T))$, with $\operatorname{End}(T)$ the bundle of endomorphisms of the tangent bundle, $T[39,1]$. 


\section{REFERENCES}

1. M.B. Green, J.H. Schwarz and E. Witten, Superstring Theory, Cambridge University Press, 1987.

2. C.G. Callan, D. Friedan, E.J. Martinec and M.J. Perry, Nucl.Phys. B262 (1985) 593.

3. For a review see C.G. Callan and L. Thorlacius, Particles, Strings and Supernovae, Vol.2 TASI 88, eds. A. Jevicki and C.-I. Tan, World Scientific (1989).

4. R.R. Khuri, Nucl.Phys. B387 (1992) 315.

5. A. Dabholkar, G. Gibbons, J. Harvey and F.R. Ruiz, Nucl.Phys B340 (1990) 33.

6. C. Callan, J. Harvey and A. Strominger, Nucl.Phys B359(1991) 611; ibid. B367 (1991) 60.

7. M.J. Duff and J.X. Lu, Nucl.Phys. 354 (1991) 141;

A. Strominger, Nucl.Phys. B343 (1990) 167.

8. L. Alvarez-Gaumé and D.Z. Freedman, Phys.Rev. D15 (1980) 846; Comm. Math. Phys. 80 (1981) 443;

L. Alvarez-Gaumé, Nucl.Phys. 184 (1981) 180;

L. Alvarez-Gaumé and P. Ginsparg, Comm. Math. Phys. 102 (1985) 311;

C. Hull, Nucl.Phys. 260 (1985) 182.

9. E.A. Bergshoeff and M. de Roo, Nucl.Phys. B328 (1989) 439.

10. P. Candelas, G. Horowitz, A. Strominger and E. Witten, Nucl. Phis. B258 (1985) 46

11. J.M. Charap and M.J. Duff, Phys.Lett. 69B (1977) 445.

12. S.-J. Rey, Phys.Rev. D43 (1991) 526.

13. T. Buscher, Phys. Lett. 159B (1985); 194B (1987); 201B (1988). 
14. M. Roček, C. Ahn, K. Schoutens and A. Sevrin, Superspace WZW Models and Black Holes, IASSNS-HEP-91-69, Contribution to Workshop on Superstrings and Related Topics, Trieste, Italy, Aug. 8-9, 1991, hep-th/9110035.

15. E. Witten, Comm. Math. Phys. 117 (1988) 353; Supersymmetric Yang-Mills Theory on a Four-Manifolds, IASSNS-HEP-94/5.

16. S.W. Hawking and C.N. Pope, Nucl.Phys. B146 (1978) 381.

17. T. Eguchi and A.J. Hanson, Ann.Phys.120 (1979) 82.

18. For a review on gravitational instantons see: T. Eguchi, P.B. Gilkey and A.J. Hanson, Phys.Rep. 66 (1980) 213.

19. G. 't Hooft, Phys. Rev. D14 (1976) 3422.

20. A. Yaffe, Nucl. Phys. B151 (1979) 247.

21. V. Novikov, M. Shifman, A. Vainshtein and V. Zakharov, Nucl.Phys. B260 (1985) 157;

D. Amati, G.C. Rossi and G. Veneziano, Nucl.Phys. B249 (1985) 1;

I. Affleck, M. Dine and N. Seiberg, Nucl.Phys. B256 (1985) 557;

D. Amati, K. Konishi, Y. Meurice, G.C. Rossi and G. Veneziano, Phys.Rep. $162(1988) 169$.

22. E. Witten, Nucl.Phys. B185 (1981) 513.

23. K. Konishi, N. Magnoli and H. Panagopoulos, Nucl.Phys. B309 (1988) 201; ibid. B323 (1989) 441.

24. K. Konishi, Phys.Lett. B135 (1984) 439.

25. P.K. Townsend and P. van Niewenhuizen, Phys.Rev.19D (1979) 3592.

26. S.-J. Rey and T.R. Taylor, Phys.Rev.Lett. 71 (1993) 1132.

27. M. Bianchi, F. Fucito, M. Martellini and G.C. Rossi, in preparation.

28. G.F. Chapline and N.S. Manton, Phys.Lett. 120B (1983) 105.

29. E.S. Fradkin and A.A. Tseytlin, Phys. Lett. 158B (1985) 316. 
30. Euclidean Quantum Gravity, eds. G. Gibbons and S.W. Hawking, World Scientific Co., 1993.

31. G.W. Gibbons, S.W. Hawking and M.J. Perry, Nucl. Phys. B138 (1978) 141 ;

G.W. Gibbons and C.N. Pope, Comm. Math. Phys. 66 (1979) 267.

32. N.J. Hitchin, A. Karlhede, U. Lindström and M. Roček, Comm.Math.Phys. 108 (1987) 535.

33. E. Alvarez, L. Alvarez-Gaumé and Y. Lozano, On Non-Abelian Duality, CERN-TH-7204/94.

34. D. Anselmi, M. Billo', P. Fre', L. Girardello and A. Zaffaroni, ALE Manifolds and Conformal Field Theories, SISSA/44/92/EP, hep-th/9304135

35. M.K. Prasad, Phys.Lett. 83B (1979) 310.

36. A.J. Hanson and H. Römer, Phys.Lett.80B (1978) 58;

S.M. Christensen and M.J. Duff, Nucl.Phys. B154 (1979) 301;

H. Römer, Phys.Lett.83B (1979) 172.

37. C.N. Pope, J.Phys. A14 (1981) L133.

38. C.N. Pope and A.L. Yuille, Phys.Lett. 78B (1978) 424;

C.N. Pope, Nucl.Phys. B141 (1978) 432.

39. E. Witten, Nucl.Phys. B268 (1986) 79.

40. N. Seiberg and E. Witten, hep-th/9407087, RU-94-52, IAS-94-43

41. J.N. Goldberg, A.J. MacFarlane, E.T. Newman, F. Rohrlich and C.G. Sudarshan, J. Math. Phys. 8 (1967) 2155;

E.T. Newman and R. Penrose, J. Math. Phys.7 (1966) 863;

E.D. Fackerell and R.G. Crossman, J. Math. Phys.18 (1977) 1849.

42. N.J. Hitchin, Math.Proc.Camb.Phil.Soc. 85 (1979) 465;

P.B. Kronheimer, J. Diff. Geom. 29 (1989) 665; ibid. 29 (1989) 685. 
43. B.R. Greene, A. Shapere, C. Vafa and S.-T. Yau, Nucl.Phys. B337 (1990) 1. 